\title{
Regulating trained immunity with nanomedicine
}

\author{
Mandy M. T. van Leent ${ }^{1,2,3}$, Bram Priem ${ }^{1,2,4}$, David P. Schrijver ${ }^{5}$, Anne de Dreu (1) ${ }^{5}$, \\ Stijn R. J. Hofstraat ${ }^{5}$, Robby Zwolsman ${ }^{5}$, Thijs J. Beldman ${ }^{6}$, Mihai G. Netea ${ }^{6,7,8 凶}$ \\ and Willem J. M. Mulder $\mathbb{1}^{5,6 凶}$
}

Abstract | Trained immunity refers to a hyperresponsive functional state of the innate immune system, which is induced by certain stimuli, such as infections or vaccination. Trained immunity plays a key part in a variety of diseases, including cancer and inflammation, and is regulated through epigenetic and metabolic reprogramming of haematopoietic stem and progenitor cells in the bone marrow, giving rise to hyperactive myeloid cells. Nanomaterials inherently interact with phagocytic myeloid cells and are thus ideal platforms with which to regulate trained immunity. In this Review, we discuss the key pathways of trained immunity and investigate nanomedicine strategies to therapeutically regulate trained immunity. Nanomedicine can be applied not only to induce trained immunity to treat cancer or to enhance resistance to infections, but also to manage hyperinflammation and maladaptive trained immunity in a variety of clinical scenarios. We conclude with an outlook to future possibilities and some remaining challenges for nanomedicine approaches in trained immunity regulation.

The clinical translation of the COVID-19 mRNA vaccines has expedited a paradigm shift in the field of nanomedicine, which has been developed from twenty-first century immuno-oncology successes. Rather than focusing on drug delivery approaches designed to evade the immune system, the field has started to embrace an engagement of the innate immune system - the body's first line of defence against invading pathogens through nanoparticle-phagocyte interactions ${ }^{1}$. Here, we refer to a specific application of nanomedicine involving the systemic administration of nanomaterials (size range of $10 \mathrm{~nm}$ to $250 \mathrm{~nm}$ ) to achieve efficient haematopoietic system engagement and therapeutic innate immune regulation. It has long been assumed that immune memory is located only in our immune system's adaptive arm, allowing effective and antigen-specific immune responses against re-encounters. However, this paradigm has been challenged by increasing evidence for the existence of a primitive innate immune memory, referred to as trained immunity ${ }^{2,3}$. Upon exposure to a pathogen-associated molecular pattern (PAMP), innate immune cells (and, in particular, myeloid cells) become more responsive to subsequent unrelated infections ${ }^{4}$. Trained immunity, which was first described in individuals who had been vaccinated with Bacillus Calmette-Guérin (BCG) ${ }^{5}$ (BOX 1), is one of the fastest growing fields in immunology.

Nanomedicine holds great promise for innate immune regulation, and may work well in conjunction with adaptive immune system-directed immunotherapeutic approaches - such as checkpoint blockade ${ }^{6}$ and chimeric antigen receptor $\mathrm{T}$ cells, for example through $\mathrm{T}$ cell co-stimulation ${ }^{7}$ or by modifying the tumour microenvironment ${ }^{6}$. In particular, trained immunity induction has the potential to mature into a compelling therapeutic approach. The use of microbial compounds to boost an immune response was explored in the late 1800s by William Coley ${ }^{8}$, who observed spontaneous tumour remission in a cancer patient with a concurrent Streptococcus pyogenes infection, which led him to hypothesize that deliberately injecting patients with this microbe could induce tumour remission ${ }^{9}$. However, owing to variable results and the obvious risk of life-threatening infections, the method quickly fell victim to the successes of radiation and chemotherapy ${ }^{10}$. Nevertheless, Coley's work inspired the search for alternative applications of boosting innate immunity using microbe-derived therapeutics. In the 1970s, the BCG vaccine, which was originally developed as a vaccine against tuberculosis, was approved for treating high-risk non-muscle-invasive bladder cancer ${ }^{11}$ (BOX 1). This specific application is safe because the urothelium does not absorb BCG and harmful systemic uptake or bacteraemia are prevented. However, to take full advantage of the therapeutic potential of trained immunity, innovative means of safely inducing it must be developed. Trained immunity owes its longevity to progenitors in 


\section{Box 1 | BCG vaccination}

The field of trained immunity originates from research with the Bacillus CalmetteGuérin (BCG) vaccine $\mathrm{e}^{178,179}$, which was developed in the 1920 s to enhance protection against tuberculosis. The heterologous features of this vaccine were recognized by Holmgren, who first adopted BCG as a stomach cancer treatment in 1935 (REF. ${ }^{180}$ ). BCG has been trialled as a treatment against a range of cancers, including tumours of the kidney, colon, prostate, bladder, lung and skin, as well as haematological malignancies ${ }^{181-186}$. Owing to the risks associated with systemic administration, BCG is approved by the US Food and Drug Administration (FDA) only for treating non-invasive bladder cancer, for which it is intravesically administered and kept isolated from the rest of the body. BCG is also applied to enhance protection against infections other than tuberculosis. Its nonspecific protective effects are attributed to metabolic and epigenetic reprogramming of myeloid cells and their progenitors, that is, trained immunity ${ }^{3,5}$. The nonspecific benefits of regulating trained immunity have recently been demonstrated ${ }^{120}$ in a double-blind, randomized trial. In elderly individuals, BCG vaccination reduced viral respiratory tract infections by $80 \%$ over a period of 12 months, as compared to placebo-vaccinated individuals.

During the COVID-19 pandemic, numerous BCG trials have been initiated (NCT04384549, NCT04659941, NCT04461379), with the goal of providing increased protection against SARS-CoV-2 infection to the vulnerable and frontline workers ${ }^{115}$. There is evidence that the age-dependency in COVID-19 severity may be related to innate immune responses ${ }^{187}$. In children and young adults, the innate immune responses to a SARS-CoV-2 infection are faster and much stronger, whereas in the elderly, these responses are muted by decades of pathogen exposure. Therefore, future pandemics could be mitigated by bridge-vaccination with trained immunity-inducing vaccines, such as BCG, MTBVAC (Mycobacterium tuberculosis vaccine), the measles vaccine, the smallpox vaccine or the oral polio vaccine ${ }^{188-192}$. These vaccines may induce protective innate immune responses for rapid viral clearance, similar to the protection children and young adults experience, limiting the detrimental damage of long-lasting infections.

the bone marrow ${ }^{12}$, which undergo metabolic and epigenetic rewiring to continue producing 'trained' myeloid cells ${ }^{6}$, rendering these cells hyperresponsive. Therefore, technologies must be developed that directly engage myeloid progenitors ${ }^{13}$.

Nanomaterials inherently interact with phagocytic myeloid cells, and are thus ideal tools to regulate trained immunity by delivering drugs to myeloid cells and their progenitors in the bone marrow, including small molecules $^{6,7}$, polymers $^{14}$, RNA therapeutics ${ }^{15}$ and immunoregulatory proteins ${ }^{16}$. Nanomedicine-based activation of trained immunity will find use in cancer treatment and will increase resistance to infection. Conversely, inhibition of trained immunity has therapeutic benefits in conditions that are characterized by an exacerbated

\footnotetext{
Author addresses

${ }^{1}$ BioMedical Engineering and Imaging Institute, Icahn School of Medicine at Mount Sinai, New York, NY, USA.

2Department of Diagnostic, Molecular and Interventional Radiology, Icahn School of Medicine at Mount Sinai, New York, NY, USA.

${ }^{3}$ Cardiovascular Research Institute, Icahn School of Medicine at Mount Sinai, New York, NY, USA.

${ }^{4}$ Angiogenesis Laboratory, Amsterdam UMC, Cancer Center Amsterdam, Amsterdam, Netherlands.

${ }^{5}$ Laboratory of Chemical Biology, Department of Biomedical Engineering, Eindhoven University of Technology, Eindhoven, Netherlands.

${ }^{6}$ Department of Internal Medicine and Radboud Center for Infectious diseases (RCl), Radboud University Nijmegen Medical Centre, Nijmegen, Netherlands.

${ }^{7}$ Radboud Institute for Molecular Life Sciences, Radboud University Medical Centre, Nijmegen, Netherlands.

${ }^{8}$ Department for Genomics \& Immunoregulation, Life and Medical Sciences Institute (LIMES), University of Bonn, Bonn, Germany.
}

immune response, such as autoimmune disorders, cardiovascular disease, sepsis, transplant rejection and acute respiratory distress syndrome.

\section{The innate immune system}

The immune system of vertebrates can be divided into an innate arm and an adaptive arm. The innate arm consists of external defences (physical barriers such as the skin and stomach acid) and internal defences ${ }^{17}$. Internal defences are activated by pattern recognition receptors of innate immune cells. These receptors can sense PAMPs in case of infection, or damage-associated molecular patterns (DAMPs), which are non-microbial triggers. Once activated, innate immune cells set in motion the complement system, which ultimately results in the killing of pathogens or of infected or malignant cells through phagocytosis. Phagocytosis is a type of endocytosis - a process by which a cell engulfs a particle. Although most cells are capable of phagocytosis, it is the main function of some cells, including myeloid innate immune cells, such as monocytes, macrophages, dendritic cells and neutrophils ${ }^{18}$. Therefore, innate immunity provides a nonspecific, efficient and rapid reaction to protect the host. Certain innate immune cells, such as dendritic cells and macrophages, can also initiate an antigen-specific adaptive immune response through a combination of antigen presentation (signal 1), co-stimulation (signal 2) and cytokine regulation (signal 3), which takes several days to develop ${ }^{19-22}$.

The immune cells of our host defence system are the result of haematopoiesis, that is, the highly dynamic process of blood cell production, which involves the generation of leukocytes from haematopoietic stem and progenitor cells (HSPCs) residing in the bone marrow ${ }^{23}$. Innate immune cells include all leukocytes from the myeloid lineage, such as monocytes, macrophages and granulocytes, as well as some leukocytes from the lymphoid lineage. Besides myeloid cells, HSPCs also give rise to $\mathrm{T}$ and $\mathrm{B}$ lymphocytes, which are part of the adaptive immune system, and non-leukocytes, including thrombocytes and erythrocytes ${ }^{24}$.

A growing body of evidence indicates that the innate immune system displays memory characteristics ${ }^{2}$. DAMPs and PAMPs can induce functional reprogramming of innate immune cells, resulting in an altered response towards a subsequent challenge with a related or unrelated stimulus. This concept is referred to as trained immunity, in which 'trained' cells display a stronger response upon secondary stimulation, whereas the terms 'tolerance' or 'immune paralysis' describe muted immune responses ${ }^{3}$. Memory function of the adaptive immune system is mediated by gene recombination; by contrast, trained innate immunity relies on epigenetic reprogramming of transcriptional pathways. Shifts in cellular metabolism underlie these epigenetic changes. Myeloid-derived monocytes and macrophages were first found to display trained and tolerant phenotypes ${ }^{25}$. We now know that neutrophils, natural killer (NK) cells and non-leukocytes, such as epidermal cells, also display innate immune memory features ${ }^{26-28}$. Trained immunity can last for months to a few years and is systemically regulated through myelopoiesis. Indeed, 
certain HSPC populations undergo epigenetic rewiring, giving rise to 'trained' myeloid cells ${ }^{29}$.

\section{Targeting trained immunity}

Trained immunity can be targeted at multiple levels, which should all be considered in the design of nanomedicines, including whole-body level, system or organ-system level, cellular level and subcellular level. On a whole-body level, myeloid cell-rich haematopoietic organs, such as the spleen and bone marrow, are important targets (FIG. 1a). HSPCs reside in the bone marrow, where they can be transcriptionally reprogrammed, for example, upon BCG vaccination, resulting in a persistent trained phenotype ${ }^{29}$. Therefore, nanomaterials that can induce trained immunity in the bone marrow are particularly interesting.

\section{The haematopoietic system}

Monocytes and macrophages have pivotal roles in innate immune memory, and the molecular mechanisms underlying trained immunity in these cells have been thoroughly investigated ${ }^{25,30}$ (FIG. 1 b). Moreover, these cells are professional phagocytes, guaranteeing the uptake of nanomaterials ${ }^{31}$. Immune memory has also been described in neutrophils, which make up a large proportion of myeloid cells. BCG-induced and $\beta$-glucan-induced trained immunity further increases the generation of these cells (granulopoiesis) ${ }^{12,32}$, which can result in anti-tumour and antimicrobial activity ${ }^{27,33}$, highlighting the therapeutic potential of neutrophil targeting. Dendritic cells, which also exhibit immune memory ${ }^{34}$, can modulate both the innate and adaptive arms of the immune system ${ }^{35}$, making them a promising trained immunity target. Furthermore, non-myeloid innate immune cells, such as NK cells and other innate lymphoid cells, can undergo antigen-independent reprogramming, displaying trained immunity features ${ }^{36-40}$. However, these cells do not possess phagocytic capacities, so an active targeting approach would be required, for example, through surface functionalization of nanomaterials with ligands specific to CD56. Targeting mature innate immune cells has the advantage of delivering immediate effects; however, more substantial therapeutic benefits may be achieved by HSPC reprogramming, because the durability of trained immunity relies on these cells ${ }^{12,29}$. Although HSPCs make up only a tiny proportion of immune cells, they give rise to the entire immune landscape and are much longer-lived than monocytes or neutrophils ${ }^{24}$.

\section{Cellular regulation}

On a cellular level, a selection of receptors can be targeted to induce or inhibit trained immunity (FIG. 1 C; TABLE 1). In particular, pattern recognition receptors for PAMPs have been extensively studied, because trained immunity was first described as an altered immune response after pathogen encounters. For example, the C-type lectin receptor Dectin-1 is involved in antifungal immunity and binds various $\beta$-glucans, each resulting in different downstream effects ${ }^{41}$. $\beta$-Glucan derived from the cell wall of Candida albicans induces trained immunity ${ }^{25}$, whereas laminarin, a mixture of short-chain $\beta$-glucans from seaweeds, competitively inhibits agonistic $\beta$-glucan binding to dectin 1 (REFS ${ }^{41,42}$ ). These findings

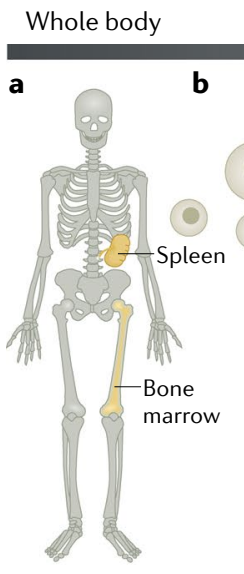

Fig. 1 | Trained immunity targeting levels. a | The spleen and bone marrow are important target organs, because they produce and contain large numbers of innate immune cells. $\mathbf{b}$ | Mature innate immune cells (innate lymphocytes, dendritic cells, monocytes, neutrophils and macrophages) and haematopoietic stem and progenitor cells can be targeted to prevent or enhance trained immunity. c| Pattern recognition receptors play an important part in trained immunity. Examples include dectin 1, Toll-like receptor 4 (TLR4) and nucleotide-binding oligomerization domain-containing protein 2 (NOD2). These receptors recognize pathogen-associated molecular patterns (PAMPs) and damage-associated molecular patterns (DAMPs). CD131 is the common $\beta$-subunit of granulocyte-macrophage colony-stimulating factor (GM-CSF) and interleukin-3 (IL-3) receptors. The IL-1 receptor (IL-1R) binds to IL-1 $\beta$. Insulin-like growth factor 1 receptor (IGF1R) recognizes extracellular mevalonate. $\mathbf{d}$ | Intracellular metabolic pathways that can be targeted include glycolysis ${ }^{4}$ (through interference with glycolytic enzymes or indirect through mechanistic target of rapamycin (mTOR) inhibition), cholesterol metabolism ${ }^{54}$ (by targeting HMG CoA reductase), glutaminolysis ${ }^{60}$ (through glutaminase inhibitors) and the tricarboxylic acid cycle (TCA) cycle (for example, by restricting succinate oxidation). e | H3K4me3 and K3K27ac are hallmark epigenetic signatures of trained immunity, which can be modified by targeting lysine demethylase (KDM), lysine methyltransferase (KMT), histone deacetylase (HDAC) and histone acetyltransferase (HAT) activity. Immune gene-priming long non-coding RNAs (IPLs) facilitate trimethylation of cytokine promotors ${ }^{66}$. PI3K, phosphatidylinositol 3-kinase. 
Table 1 | Trained immunity induction

\begin{tabular}{|c|c|c|}
\hline Receptor & DAMP or PAMP & Refs \\
\hline Dectin 1 & $\beta$-Glucan & \\
\hline NOD2/NOD1 & MTP & 6,47 \\
\hline TLR4 & $\begin{array}{l}\text { Very low concentrations of LPS } \\
\text { oxLDL } \\
\text { Lipoprotein(a) } \\
\text { Urate } \\
\text { Vimentin } \\
\text { HMGB1 }\end{array}$ & $7,48-5$ \\
\hline TLR2 & Low concentrations of Pam3Cys & 174 \\
\hline TLR3 & Poly I:C & 175 \\
\hline IGF1R & Mevalonate & 54 \\
\hline \multicolumn{3}{|c|}{$\begin{array}{l}\text { DAMP, damage-associated molecular pattern; HMGB1, high } \\
\text { mobility group box protein 1; IGF, insulin-like growth factor; } \\
\text { LPS, lipopolysaccharide; MTP, muramyl tripeptide; NOD, } \\
\text { nucleotide-binding oligomerization domain-containing } \\
\text { protein; oxLDL, oxidized low-density lipoprotein; PAMP, } \\
\text { pathogen-associated molecular pattern; poly I:C, } \\
\text { polyinosinic:polycytidylic acid; TLR, Toll-like receptor. }\end{array}$} \\
\hline
\end{tabular}

create opportunities for developing $\beta$-glucan nanoformulations. Activated Dectin-1 subsequently triggers phosphoinositide 3-kinase (PI3K) signalling, which is negatively regulated by $\mathrm{SH} 2$ domain-containing inositol $5^{\prime}$-phosphatase 1 (SHIP1) ${ }^{43}$ (TABLE 2). Pharmacological inhibition of SHIP1 by $3 a$-aminocholestane enhances Candida albicans-induced trained immunity ${ }^{44}$. SHIP1 is also the primary target of microRNA-155 $(\mathrm{miR}-155)^{45}$, and miR-155 overexpression leads to repression of SHIP1 and subsequent PI3K/Akt activation. Therefore, miR-155-containing polymeric nanoparticle formulations ${ }^{46}$ may allow trained immunity amplification.

The nucleotide-binding oligomerization domaincontaining protein 2 (NOD2) is a pattern recognition receptor involved in trained immunity induction. This intracellular receptor recognizes bacterial peptidoglycans $s^{47}$ by binding to muramyl dipeptide (MDP), which is present on Gram-positive and -negative bacteria. For example, the BCG vaccine induces trained immunity through NOD2 activation ${ }^{5}$. In a murine melanoma model, pharmacological stimulation of NOD2 by nanobiologics (which are apolipoprotein A1-based nanomaterials that are exclusively composed of natural molecular building blocks, decorated with muramyl tripeptide (MTP)) induces trained immunity and inhibits cancer growth ${ }^{6}$. Similarly, Toll-like receptor 4 (TLR4) can be targeted for trained immunity modulation. DAMPs, such as oxidized low-density lipoprotein (oxLDL), lipoprotein(a), urate, vimentin and high-mobility group box 1 (HMGB1), are endogenous stimuli that induce trained immunity ${ }^{7,48-50}$ and that can be recognized by TLR4 (REF. ${ }^{51}$ ). This receptor can be blocked by the small-molecule inhibitor TAK242

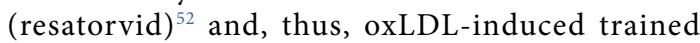
immunity can be suppressed by resatorvid ${ }^{53}$.

In addition, cytokines and metabolites play an important part in innate immune memory regulation, making them attractive targets for immunotherapy. Upon trained immunity induction, granulocyte-macrophage colony-stimulating factor (GM-CSF) signalling enhances progenitor proliferation in the bone marrow, and pharmacologic inhibition of GM-CSF counteracts this process ${ }^{12}$. A similar effect can be established through pharmacologic inhibition of interleukin-1 (IL-1) by the IL-1 receptor antagonist anakinra ${ }^{12}$. The metabolite mevalonate binds and activates the extracellular insulin-like growth factor 1 receptor (IGF1R), which leads to downstream mechanistic target of rapamycin (mTOR) signalling and upregulated glycolysis, resulting in histone modifications of pro-inflammatory enzymes and a trained immunity phenotype ${ }^{54}$. This process can be prevented by an inhibitor or antibody targeted to IGF1R ${ }^{54}$.

\section{Metabolic targets}

Receptor binding often results in the activation of specific metabolic pathways (FIG. 1d), which can, in turn, also be a target for trained immunity modulation. For example, induction of trained immunity strongly increases glycolysis, which can be reduced by inhibition of hexokinases with 2-deoxy-D-glucose ${ }^{55}$. Glycolysis is not only controlled by enzyme activity, but also by mTOR signalling, and can therefore be inhibited through pharmacologic mTOR interference with rapamycin, metformin or ascorbate ${ }^{4}$. For example, nanoformulations containing rapamycin inhibit trained immunity and prolong graft survival in a murine heart allograft model ${ }^{7}$. Interference with cholesterol metabolism, for example, through 3-hydroxy-3-methyl-glutaryl-coenzyme A (HMG-CoA) reductase inhibition with statins ${ }^{54}$, reduces mevalonate accumulation and subsequent IGF1R and mTOR activation. The lipophilic nature of statins, such as fluvastatin and simvastatin, facilitates their incorporation into lipid-based nanoformulations ${ }^{56}$. For example, simvastatin-loaded reconstituted high-density lipoproteins (S-HDL) are taken up by aortic plaque macrophages in atherosclerosis-prone mice ${ }^{57-59}$. Interestingly, treatment with S-HDL reduces atherosclerotic plaque inflammation in mice and translational animal models ${ }^{59}$.

In addition to glycolysis and cholesterol synthesis, glutaminolysis is upregulated in trained immunity. Glutamine is processed to glutamate, which is subsequently converted to $a$-ketoglutarate or succinate, which are metabolites in the tricarboxylic acid (TCA) cycle. Succinate oxidation results in the accumulation of fumarate, which inhibits histone demethylases, resulting in increased histone methylation of pro-inflammatory cytokines ${ }^{60}$. This process can be mitigated by using bis-2-(5-phenylacetamido-1,2,4-thiadiazol-2-yl)ethyl sulfide (BPTES), a glutaminase inhibitor ${ }^{60}$. Trained immunity can also be modulated by directly targeting the TCA cycle, for example, with dimethyl malonate, which is an inhibitor of succinate oxidation ${ }^{61}$ that can be incorporated into a nanobiologic platform ${ }^{62}$. By inhibiting succinate oxidation, dimethyl malonate prevents fumarate accumulation and its downstream epigenetic effects.

\section{Epigenetic targets}

The epigenome forms the molecular basis of trained immunity and can be modified by modulating the activity of epigenetic enzymes. Various types of cancer 
also display maladaptive epigenetic rewiring, and several epigenetic inhibitors have already been approved or tested in clinical trials for the treatment of haematologic malignancies and solid tumours ${ }^{63}$. Nanomedicine can be applied to redirect epigenetic drugs to innate immune cells for trained immunity modulation, allowing fine-tuning of the biodistribution and cellular uptake of drugs.

A specific epigenetic hallmark of trained immunity is acetylation of histone 3 lysine 27 (H3K27ac) at distal enhancers ${ }^{64}$ (FIG. 1e). Histone acetylation renders chromatin more accessible, facilitating gene transcription. During induction of trained immunity, these histone modifications are typically found on genes encoding pro-inflammatory cytokines and metabolic enzymes. Acetylation levels are dependent on the presence of histone acetyltransferases and deacetylases, which can be used as therapeutic targets. For example, (-)-epigallocatechin-3-gallate (EGCG), a histone acetyltransferase inhibitor, inhibits histone modifications associated with $\beta$-glucan-induced trained immunity ${ }^{44}$. In contrast to histone acetylation, histone methylation can be either repressive or activating. Histone methyltransferases can be inhibited with 50-deoxy-50-(methylthio)adenosine (MTA $)^{25}$. Methylation of DNA, which is associated with repressed gene transcription, is regulated by DNA methyltransferases, which can be inhibited by azacitidine encapsulated in a polylactide-co-glycolide (PLGA)-polyethylene glycol (PEG) nanoscale delivery system. Here, incorporation into the nanomaterial improves the therapeutic effect by enhancing drug

Table 2 | Trained immunity regulation

\begin{tabular}{|c|c|c|c|c|}
\hline Pathway & Target & Drug & Result & Ref. \\
\hline \multirow{3}{*}{$\begin{array}{l}\mathrm{PI} 3 \mathrm{~K} / \mathrm{AKT} / \\
\mathrm{mTOR} / \mathrm{HIF} 1 \mathrm{a}\end{array}$} & \multirow[t]{3}{*}{ SHIP1 } & 3a-Aminocholestane & Enhancement & 44 \\
\hline & & miR-155 & Enhancement & 45 \\
\hline & & Anakinra & Inhibition & 12 \\
\hline $\begin{array}{l}\text { Cholesterol } \\
\text { synthesis }\end{array}$ & HMG-CoA reductase & Statins & Inhibition & 54 \\
\hline \multirow[t]{4}{*}{ Glycolysis } & Hexokinases & 2-Deoxy-D-glucose & Inhibition & 55 \\
\hline & \multirow[t]{3}{*}{ mTOR } & Rapamycin & Inhibition & 4 \\
\hline & & Metformin & Inhibition & 4 \\
\hline & & Ascorbate & Inhibition & 4 \\
\hline \multirow[t]{2}{*}{ Glutaminolysis } & Glutaminase & BPTES & Inhibition & 60 \\
\hline & Succinate oxidation & Dimethyl malonate & Inhibition & 61 \\
\hline $\begin{array}{l}\text { Histone } \\
\text { acetylation }\end{array}$ & $\begin{array}{l}\text { Histone } \\
\text { acetyltransferase }\end{array}$ & EGCG & Inhibition & 44 \\
\hline \multirow[t]{2}{*}{$\begin{array}{l}\text { Histone } \\
\text { methylation }\end{array}$} & $\begin{array}{l}\text { Histone } \\
\text { methyltransferases } \\
(\mathrm{H} 3 \mathrm{~K} 4)\end{array}$ & MTA & Inhibition & 25 \\
\hline & $\begin{array}{l}\text { Histone } \\
\text { methyltransferases } \\
\text { (H3K9) }\end{array}$ & BIX-01294 & Enhancement & 176 \\
\hline $\begin{array}{l}\text { DNA } \\
\text { methylation }\end{array}$ & $\begin{array}{l}\text { DNA } \\
\text { methyltransferases }\end{array}$ & Azacitidine & Inhibition & 65 \\
\hline
\end{tabular}

BPTES, bis-2-(5-phenylacetamido-1,2,4-thiadiazol-2-yl)ethyl sulfide; EGCG, epigallocatechin gallate; HIF1 $\alpha$, hypoxia inducible factor $1 \alpha$; HMG-CoA, 3-hydroxy-3-methylglutaryl-CoA; MTA, methylthioadenosine; mTOR, mechanistic target of rapamycin; PI3K, phosphoinositide 3-kinase; SHIP1, SH2 domain-containing inositol 5'-phosphatase 1. stability and increasing cellular uptake ${ }^{65}$, demonstrating the potential of nanoformulations to increase epigenetic drug efficacy.

Histone modifications can also be altered by long non-coding RNA (lncRNA). Upon $\beta$-glucan stimulation, a specific class of lncRNAs, called 'immune gene-priming lncRNAs' (IPLs), are upregulated, facilitating $\mathrm{H} 3 \mathrm{~K} 4$ trimethylation of cytokine promotors ${ }^{66}$. Here, topologically associated domains and the specific 3D structure of IncRNAs allow interaction with multiple innate immune genes ${ }^{67}$. The activity of UMLILO, a prototype IPL, can be inhibited by siRNA ${ }^{66}$.

\section{The in vivo behaviour of nanomaterials}

Nanomaterials can interact with the haematopoietic system, which is responsible for leukocyte production, and with the mononuclear phagocyte system, which can be exploited in the regulation of trained immunity. In particular, the bone marrow, which has rarely been the target tissue in nanomedicine studies, is important in trained immunity. Although uptake of nanoliposomes in the bone marrow has previously been reported ${ }^{68}$, targeting the bone marrow for immune regulation by nanomedicine has only recently been explored ${ }^{6,69}$. The quest to improve drug delivery by increasing the percentage of injected drug dose at the site of action upon intravenous administration has long been central to the nanomedicine community, particularly to increase drug deposition in cancerous and inflammatory lesions. This approach requires the nanodrugs to have a long circulation half-life, which can be challenging to achieve with nanomaterials, because they are usually rapidly eliminated through complement activation and phagocytes. The blood circulation time of nanomaterials can be increased through surface coating, for example, the coating of liposomes with hydrophilic polymers, such as $\mathrm{PEG}^{70}$, with an optimal PEG density of approximately $10 \mathrm{~mol} \%$ (we note that very high PEG densities decrease circulation half-lives $)^{70}$. Typically, PEGylation is achieved by formulating self-assembled nanoparticles that include PEG-functionalized phospholipids ${ }^{71}$ or polymers $^{72}$. Alternatively, nanocrystalline materials, such as gold ${ }^{73}$, iron oxide ${ }^{74}$ or semiconductor nanocrystals ${ }^{75}$, can be applied to the material post-synthesis.

\section{The mononuclear phagocyte system and nanoparticle clearance}

Drug delivery to lesions relies on high vascular permeability and long blood circulation half-lives, which can be achieved by avoiding premature elimination by the mononuclear phagocyte system. By contrast, nanomedicines intended to regulate trained immunity require efficient interaction with myeloid cells. Therefore, the affinity of nanomaterials for phagocytes should not be prevented, but rather exploited, which necessitates in-depth knowledge of the in vivo behaviour of nanomaterials. Most nanomedicine platforms are self-assembled systems based on lipid nanoparticles comprised of phospholipids and/or mixtures of other fatty molecules, or polymeric nanoparticles, which are typically built from block copolymers. The therapeutic payload of small molecules, RNA or proteins, for example, is usually integrated by 
a self-assembly process. The in vivo behaviour of such platforms is typically assessed by investigating a single nanoparticle component, that is, either the nanomaterial or the therapeutic payload. Although different components can be simultaneously labelled, for example, by using different radioisotopes or fluorophores ${ }^{56}$ for nuclear or optical detection, respectively, nanoparticle integrity or the exchange of components with blood constituents cannot be studied using standard techniques, such a gamma counting or confocal laser scanning microscopy. However, imaging methods, in combination with ex vivo approaches, have been developed to holistically study nanoparticle behaviour in preclinical models (BOX 2).

\section{The nanoparticle in vivo journey}

The in vivo fate of nanomaterials depends on material properties, such as size and surface coating, and features of the circulatory system, including endothelial permeability and neovascularization. These parameters dictate nanoparticle aggregation, organ and tissue uptake, as well

\section{Box 2 | Imaging framework}

In vivo imaging methods can support multiple aspects of nanotherapy development. In vivo imaging allows the non-invasive study of nanoparticle biodistribution and cellular interactions in vivo. For example, labelling of nanoparticles with radioisotopes, such as copper-64 $\left({ }^{64} \mathrm{Cu}\right)$ or zirconium- $89\left({ }^{89} \mathrm{Zr}\right)$, enables dynamic and quantitative positron emission tomography (PET) imaging of their biodistribution, which can also be achieved by single-photon emission computerized tomography (SPECT) imaging in combination with radioisotopes, such as technetium-99m ( $\left.{ }^{99 \mathrm{~m}} \mathrm{Tc}\right)$ and indium-111 $\left({ }^{111} \mathrm{In}\right)$. These tomographic nuclear imaging techniques can dynamically and longitudinally visualize nanoparticle distribution at the whole-body level, in a non-invasive and quantitative fashion. PET and SPECT imaging systems are typically integrated with computed tomography for anatomical reference and to allow attenuation corrections for tomographic purposes. Although technically more challenging, the combination of PET and SPECT with magnetic resonance imaging (MRI) has substantial advantages, in particular, for soft tissues with complex anatomical structures, such as the vessel wall ${ }^{59}$. A variety of methods are available to radiolabel nanomaterials ${ }^{164}$. In addition to radioisotopes, nanomaterials can be labelled with fluorophores, allowing in vivo near-infrared fluorescence (NIRF) imaging of whole-body biodistribution, and the study of nanoparticle disassembly dynamics using Förster resonance energy transfer (FRET) imaging or intravital microscopy ${ }^{5,193,194}$. The latter technique can also be applied to study nanoparticle-(immune) cell interactions in live mice ${ }^{166}$. In addition to in vivo imaging, the cellular behaviour of fluorescently labelled nanoparticles can be studied ex vivo in tissue sections by confocal microscopy and/or on (immune) cells using flow cytometry assays.

In addition to studying biodistribution and cellular specificity, imaging is also a valuable way of studying the induction or inhibition of trained immunity and inflammatory responses, as a result of pathological processes or (nano)therapeutic interventions. As trained immunity involves (rewiring of) programs at multiple levels, several imaging methods can be employed. Metabolic alterations of myeloid cells in the bone marrow are a hallmark of trained immunity. For example, glucose consumption and glutaminolysis are upregulated. These processes can be visualized by PET imaging using the radiotracers ${ }^{18} \mathrm{~F}-\mathrm{FDG}$ and ${ }^{18} \mathrm{~F}-(2 \mathrm{~S}, 4 R) 4$-fluoroglutamine $\mathrm{PET}^{195}$. Histone deacetylase PET can detect epigenetic alterations in the brain and could also be adopted for the bone marrow ${ }^{196}$. Immune cell proliferation in this organ can be studied using ${ }^{18} \mathrm{~F}$-fluorothymidine $\mathrm{PET}^{197}$. Changes in myeloid cell dynamics can be probed by ${ }^{89} \mathrm{Zr}$-labelled nanobodies targeting CD $11 b^{198,199}$. Many PET imaging methods and tracers are clinically approved and can therefore be readily implemented in clinical studies and may serve as companion diagnostics for trained-immunity-regulating nanotherapeutics. A range of modalities are also pre-clinically available to study inflammation. For example, fluorescence molecular tomography with computed tomography (FMT-CT) enables NIRF imaging of nanoparticle biodistribution ${ }^{200}$ in mice. The same method allows quantitative investigation of (macrophage) inflammation using an activatable fluorescent dye. as clearance rates and elimination pathways ${ }^{76,77}$. Upon intravenous administration, nanoparticles are transported in the blood to the right ventricle of the heart, into the lung capillaries and back to the left ventricle, before they are distributed to vital organs in the body, such as the brain, liver and kidneys, via the aorta. In general, intravenously infused nanoparticles do not extravasate from regular blood vessels lined with an impermeable endothelium or the blood-brain barrier. Therefore, in healthy individuals, nanoparticles accumulate in organs with high microvascular permeability and/or blood filtration systems, such as in the kidneys, liver and spleen. Nanoparticles can also accumulate in tumours and inflammatory lesions, including atherosclerotic plaques, which are characterized by ongoing neovascularization and increased (micro)vascular permeability ${ }^{78}$.

The key nanoparticle parameters that dictate in vivo behaviour are size, aggregation, surface charge and shape (FIG. 2). Differently sized quantum dots (semiconductor nanocrystals with size-dependent fluorescent properties) have been used to determine the size dependency of clearance, showing that nanomaterials with a hydrodynamic radius smaller than $6-8 \mathrm{~nm}$ are cleared within minutes by the kidneys and are excreted via the bladder $^{79,80}$. Kidney clearance is facilitated by the glomerular filtration membrane, which is a semipermeable membrane that filters blood. Therefore, small nanoparticles have little potential for myeloid cell targeting and trained immunity regulation. Conversely, medium-sized nanoparticles $(10-100 \mathrm{~nm})$ typically display longer blood circulation half-lives because they do not pass through the glomerular filtration barrier. Biodistribution studies show that medium-sized nanoparticles accumulate in (and are cleared by) the liver and spleen. These organs are part of the mononuclear phagocyte system, which filters toxins from the blood. Küpffer cells, which are the local macrophages of the liver, form a protective barrier through their scavenger and phagocytic functions, removing foreign debris and particles from portal blood flow through pattern recognition receptor interactions $^{76,81,82}$. In addition to Küpffer cells, B cells are involved in phagocytosis-mediated removal of certain nanomaterials, for example PEGylated quantum dots ${ }^{81}$. The spleen performs a role similar to that of the liver. Here, macrophages in the red pulp region are the primary phagocytic force driving nanoparticle removal. To achieve long blood circulation times of nanomedicines for lesion targeting, elimination by the mononuclear phagocyte system has to be prevented. Nanoparticle sizes of around 75-100 $\mathrm{nm}$ are optimal for long circulation times $^{68}$; by contrast, larger nanoparticles $(100-250 \mathrm{~nm})$ have much shorter circulation times owing to complement activation and more efficient clearance by the liver and spleen. Rapid removal can also be a result of nanoparticle aggregation upon intravenous administration, which causes the nanoparticles to become trapped in pulmonary capillaries ${ }^{83}$.

\section{Nanodelivery platforms}

Nanodelivery platforms can be applied to route different classes of therapeutic payloads to myeloid cells and their progenitors in the bone marrow and spleen. 
For example, liposomes ${ }^{84}$ have been optimized by the drug delivery community to efficiently deliver chemotherapeutics to tumours, thereby increasing the therapeutic index of drugs and reducing side effects. The blood circulation times of liposomes ${ }^{68}$ can be increased by coating with PEG and other surface-functionalization strategies ${ }^{65}$. Similarly, long-circulating polymeric nanoparticles have been designed, made of diblock copolymers from PEG and biodegradable polymers, such as PLGA. By integrating PEG, recognition by the mononuclear phagocyte system and premature removal by Küpffer cells in the liver can be avoided ${ }^{85}$. The targeting of myeloid cells, particularly macrophages, has been originally explored in the context of cardiovascular disease $^{86,87}$ and rheumatoid arthritis ${ }^{88}$. We note that studies focusing on the specific targeting of haematopoietic organs are scarce. Following the discovery that the spleen acts as a reservoir for monocytes ${ }^{89}$, a lipid nanoparticle has been designed to deliver siRNA (targeting CCR2) to the spleen and bone marrow, with the aim of preventing monocytes from migrating to sites of inflammation ${ }^{90}$. In a heart transplant mouse model, mTOR-inhibiting nanobiologic therapeutics could efficiently induce immunological tolerance by targeting myeloid cells and their progenitors in the bone marrow, resulting in prolonged allograft survival ${ }^{7}$. This approach enabled precision inhibition of inflammation. A similar nanobiologic technology could also be applied to induce trained immunity to combat cancer. Both studies $^{6,7}$ demonstrated that nanobiologic-facilitated regulation of trained immunity is caused by metabolic, epigenetic and transcriptomic changes of HSPCs in the bone marrow ${ }^{6}$.

A variety of nanomedicine platforms have been developed for drug delivery. Here, we focus on those with the highest translational potential, that is, lipid-based, polymeric, lipoprotein, viral and protein-polymer nanoparticles (FIG. 3). Lipid-based nanoparticles consist of amphiphilic (phospho)lipids, which self-aggregate or self-organize owing to the dual features of these molecules, which consist of a polar, water-soluble group attached to a water-insoluble hydrocarbon chain. The most widely studied lipid-based nanomaterials are vesicles called liposomes, which are bilayered structures encapsulating an aqueous lumen. Liposomes are highly suitable to deliver hydrophilic payloads, although their true potential has been reached with the development of different loading methods. For example, liposomal doxorubicin integrates crystals of the drug, achieving very high encapsulation efficiencies of $98 \%{ }^{91}$. Micelles are also lipid-based nanomaterials composed of spherical lipid aggregates, in which the amphiphilic lipids are organized with their apolar tails positioned in the core and the hydrophilic headgroups facing the aqueous exterior. Nanoemulsions are essentially 'swollen micelles', which additionally integrate apolar fatty molecules into their core, which is covered by a monolayer of amphiphilic lipids. Nanoemulsions and micelles are therefore suitable for the delivery of lipophilic payloads. A lipid-based platform also enabled the first approved RNA interference (RNAi) therapeutic ${ }^{92}$, and lipid nanoparticles are also integrated ${ }^{93}$ in COVID-19 mRNA vaccines ${ }^{94,95}$.
Polymeric nanoparticles composed of amphiphilic block copolymers include polymeric vesicles called polymersomes ${ }^{96}$, nanostructures with a micellar morphology ${ }^{97}$, nanoemulsions consisting of a lipophilic matrix covered by a monolayer of block copolymers ${ }^{98}$, as well as polymeric nanoparticles for RNA delivery ${ }^{99}$. Lipoprotein-derived and -inspired nanomaterials include high-density lipoprotein ${ }^{87}$, low-density lipoprotein and microemulsion-like structures, as well as a apolipoprotein A1-based nanobiologic platform ${ }^{56}$. Viral and virus-based delivery technologies have shown great potential for gene therapy and vaccination, but can also be applied for the delivery of small-molecule drugs and proteins $s^{100,101}$. Finally, nanostructures assembled from immunomodulatory proteins or polymers ${ }^{102,103}$ are made of building blocks that exhibit specific immunomodulatory features.

All of these platforms can be adjusted for the delivery of immunoregulatory payloads, albeit at different efficiencies (FIG. 3). For example, viral nanoparticles are inherently suitable for nucleic acid drug delivery, but can also be adopted for the delivery of small-molecule drugs. Liposomes are most suited for incorporating payloads in the aqueous lumen, but they can also carry lipophilic drugs in the phospholipid bilayer. It is important to harmonize the therapeutic goal, immunoregulatory payload and nanoparticle type when designing trained-immunity-regulating nanomedicines, and to take into account the drug's mode of action. For example, mRNA requires efficient cytosolic delivery to enable ribosomes to efficiently read the code and translate it into a protein. However, nanomaterials are usually endocytosed by cells and therefore, end up in cellular vesicles, called endosomes. Thus, endosomal escape is a prerequisite for and integral part of mRNA-lipid nanoparticle technologies ${ }^{104}$. There are different types of endocytosis, of which receptor-mediated (or clathrin-mediated) endocytosis is the most relevant mechanism for nanoparticle uptake. Small nanoparticles (that is, $<60 \mathrm{~nm}$ ) can also be taken up through caveolin-mediated endocytosis ${ }^{77}$. In addition, nanoparticle merging with the cell membrane, pinocytosis and contact-facilitated delivery have been proposed as mechanisms by which nanomaterials interact with cells ${ }^{105}$.

\section{Immunostimulatory polymers}

A range of immunomodulatory polymers can serve as foundational building blocks of trained immunity-inducing nanomaterials. For example, nanoparticles exclusively composed of natural polymers, such as chitosan or hyaluronan, may innately promote trained immunity ${ }^{106}$. The bacterial wall consists of peptidoglycans, which are recognized by phagocytes, inducing an immune response through NOD2 activation ${ }^{107}$. Although systemic administration of such immune-activating polymers is unattractive, nanomaterial-based formulations allow controlled exposure and reduce systemic toxicity. These immunostimulatory molecules could also be templated onto nanoparticle scaffolds or function as building blocks, either by generating copolymers or through crosslinking methods ${ }^{108}$. 
a

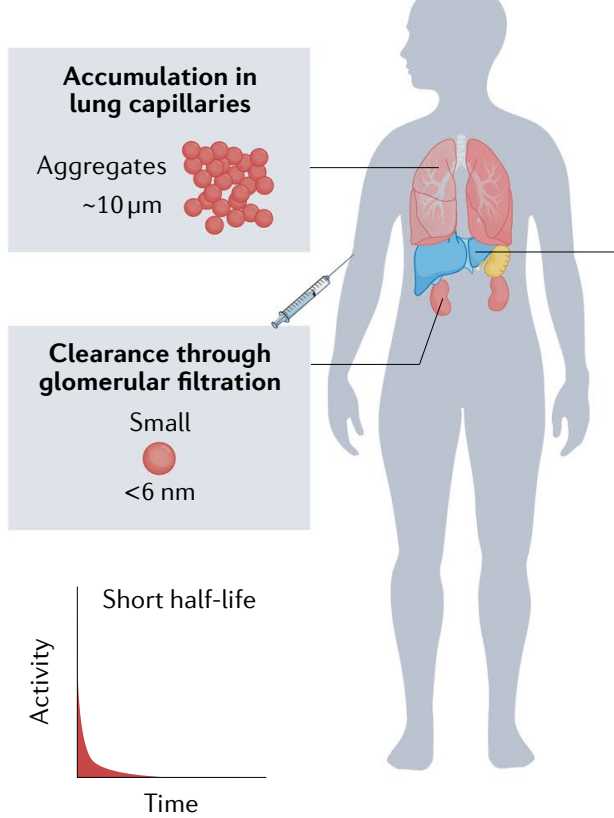

C

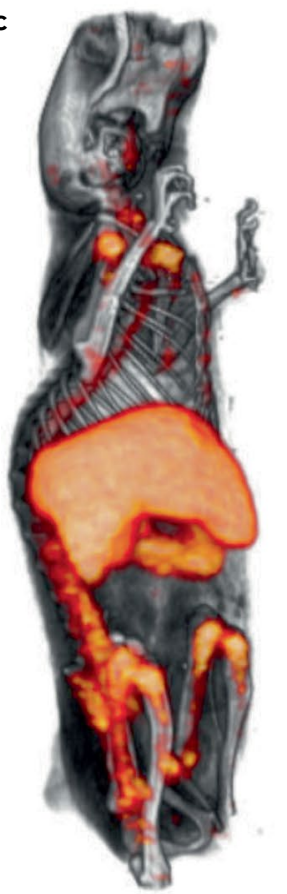

d
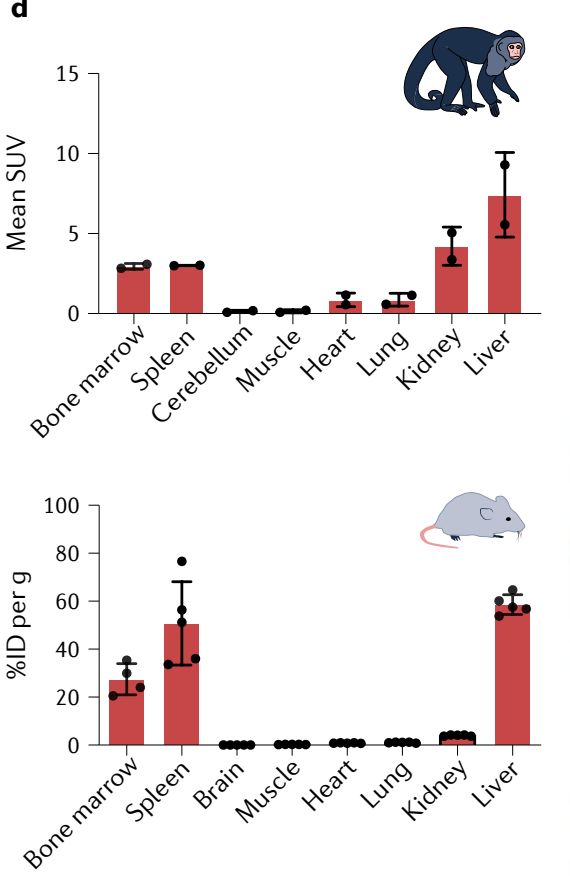
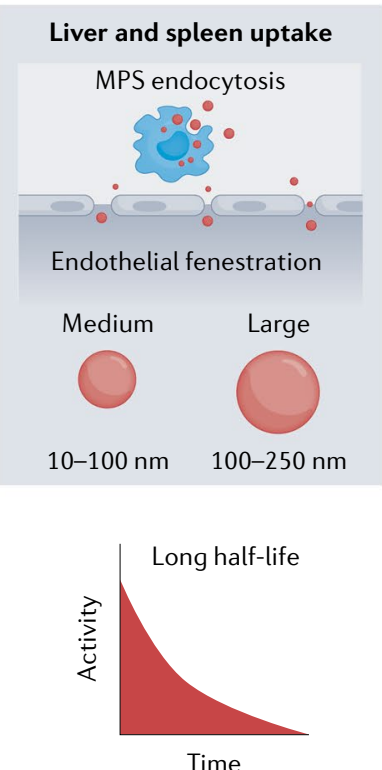
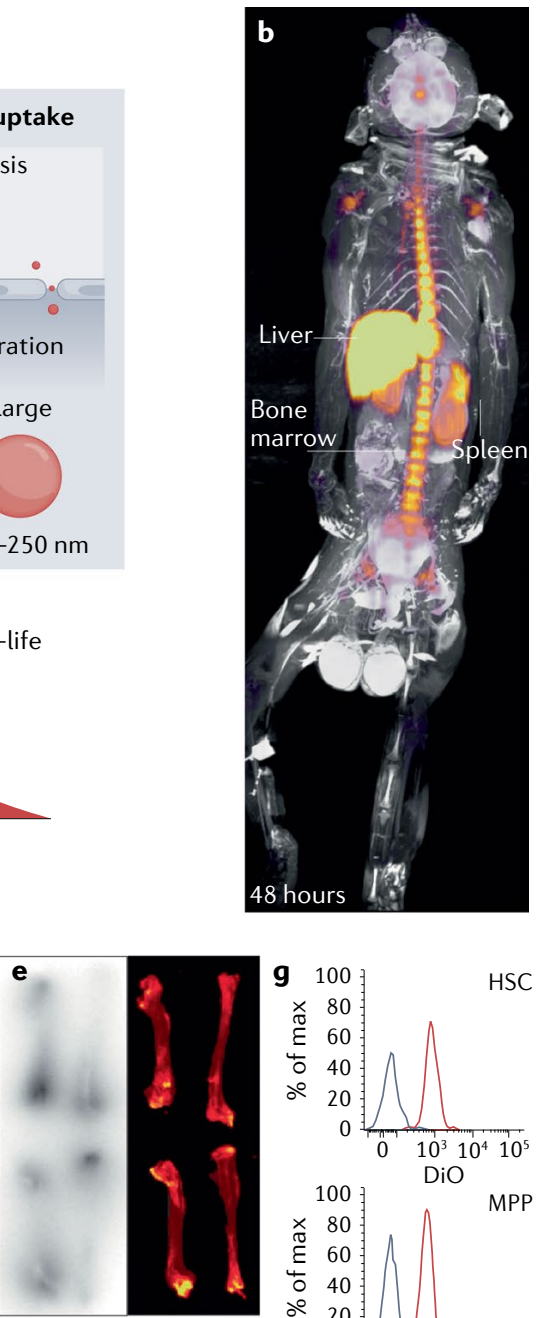

HSC

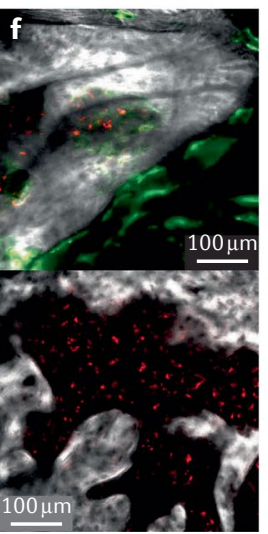

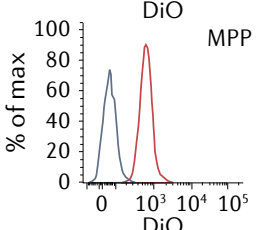

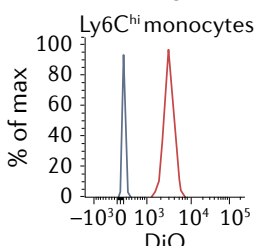
$\mathrm{DiO}$

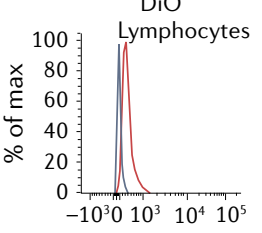

$\mathrm{DiO}$

\section{Immunoregulatory proteins}

Cytokines are key regulators of trained immunity; for example, GM-CSF and IL-1 $\beta$ promote trained immunity, and many other cytokines are currently under investigation. Cytokine-based proteins (or peptides) can be incorporated into nanomedicines; for example, polymeric nanoparticles can deliver the inflammation-resolving peptide Ac2-26 $\left(\mathrm{REFS}^{16,109}\right)$. Cytokine receptors are typically present on the cell surface, and therefore, phagocytosis (intracellular delivery) may compromise cytokine function. To prevent phagocytosis, polymeric nanoparticles can be decorated with collagen-targeting moieties to promote extravascular entrapment and avoid uptake by macrophages ${ }^{109}$. Alternatively, nanoparticles can be surface-decorated to allow cytokines to interact freely with cell surface receptors.

\section{Nucleic acid drugs}

The development of mRNA vaccines against COVID-19 has firmly established nanotechnology's utility in medicine. Lipid nanoparticle technology allowed the development of highly effective mRNA vaccines in record 
4 Fig. 2 | The journey of nanomaterials upon intravenous administration. a|Upon intravenous administration, injected nanoparticles travel to the right atrium and ventricle of the heart before entering the lung vasculature. Large aggregates are trapped in the pulmonary capillaries. Individual and unaggregated nanoparticles travel to the left side of the heart and enter the aorta. Small particles $(<6-8 \mathrm{~nm})$ are rapidly excreted through glomerular filtration in the kidneys, resulting in a short half-life in the blood. Larger formulations circulate for longer and are taken up by the liver and spleen through endocytosis by the mononuclear phagocyte system (MPS) or by endothelial fenestration. b-g | In vivo behaviour of high-density lipoprotein (HDL)-mimicking nanobiologics loaded with 10 mol\% muramyl tripeptide (MTP 10 -HDL). b | Positron emission tomography-magnetic resonance imaging (PET-MRI) scan of a non-human primate (Macaca fascicularis), 48 hours after intravenous ${ }^{89} \mathrm{Zr}$-labelled MTP 10 - $\mathrm{HDL}$ injection. There is high accumulation in the liver, spleen and bone marrow. $\mathbf{c} \mid$ Representative whole-body 3D-rendered PET-computer tomography image of a C57Bl/6 mouse, 24 hours after intravenous ${ }^{89} \mathrm{Zr}-\mathrm{MTP}_{10}-\mathrm{HDL}$ injection. Similar to the behaviour in non-human primates, ${ }^{89} \mathrm{Zr}-\mathrm{MTP}_{10}-\mathrm{HDL}$ accumulation in the liver, spleen and bone marrow are detected. $\mathbf{d} \mid$ Quantification of ${ }^{89} \mathrm{Zr}-\mathrm{MTP}{ }_{10} \mathrm{HDL}$ biodistribution in non-human primates (top, assessed by in vivo PET imaging, $n=2$ ) and mice (bottom, assessed by ex vivo gamma counting, $n=5$ ), shown as percentage injected dose (\%ID). e | Ex vivo near-infrared fluorescence (NIRF) images and autoradiography of murine femurs and tibias, 24 hours after injection of dual-labelled Dil- ${ }^{89} \mathrm{Zr}-\mathrm{MTP}_{10}-\mathrm{HDL}$. Bone marrow uptake is concentrated at the proximal and distal ends of the bone, where the red marrow is located $(n=5)$. $\mathbf{f} \mid$ Intravital microscopy image of a live mouse calvarium, 8 hours post Dil-MTP 10 -HDL administration. Fluorescein isothiocyanate (FITC)-dextran was injected intravenously to display the vasculature. $\mathrm{g}$ | Flow cytometry of murine bone marrow cells 24 hours after $\mathrm{DiO}-\mathrm{MTP}_{10}-\mathrm{HDL}$ administration (red lines) or phosphate buffered saline (PBS) control (grey lines). Representative histograms of haematopoietic stem cells (HSCs), multipotent progenitors (MPP), Ly6C ${ }^{\text {hi }}$ monocytes and lymphocytes indicate nanobiologic affinity for myeloid (progenitor) cells $(n=5)$. SUV, standardized uptake value. Panels $\mathbf{b}-\mathbf{g}$ adapted with permission from REF. ${ }^{6}$, Elsevier.

time $e^{94,95}$. Advances in lipid nanoparticle technology (in particular, the development of ionizable lipids) and in mRNA technology have forever changed the way vaccines will be developed ${ }^{110,111}$. As an alternative to lipid nanoparticles, viral vectors can be used to deliver nucleic acids, a strategy applied for the delivery of DNA in AstraZeneca's SARS-CoV-2 vaccine ${ }^{112}$. mRNA can also be delivered to myeloid cells to induce protein expression ${ }^{56,113,114}$. Similarly, other RNA-based drugs have potential for trained immunity regulation, including small interfering RNA (siRNA), microRNA (miRNA), and long non-coding RNAs (lncRNA). At varying efficiencies, all of these RNAs could be integrated into lipid or polymeric nanoparticles, for example, to silence a (hub) gene involved in crucial metabolic or epigenetic pathways involved in trained immunity using siRNA or miRNA, or to induce immunoregulatory proteins using $\mathrm{mRNA}^{15}$.

\section{Small molecules}

A range of metabolic and epigenetic pathways have been identified to regulate trained immunity (FIG. 1), and a wealth of small-molecule inhibitors have been shown to inhibit trained immunity in vitro. However, delivery of these molecules to the myeloid cell compartment will require the development of targeting strategies. Water-soluble compounds can be incorporated into the aqueous interior of liposomes or polymersomes, whereas micelles and nanoemulsions can carry lipophilic molecules. For example, prodrugs can be modularly integrated into apolipoprotein A1-based nanobiologics ${ }^{62}$, allowing greater than $80 \%$ to $90 \%$ integration of prodrug derivatives of diethylmalonate and rapamycin. Small-molecule drugs can also be integrated into viral nanoparticles. Similarly to small-molecule inhibitors, immunostimulatory molecules can be delivered by nanoparticles. Decorating the surface of nanoparticles with immunostimulatory molecules may be an efficient way to modulate trained immunity, as for nanobiologics decorated with muramyl dipeptide ${ }^{6}$.

\section{Therapeutic applications}

Nanomedicine approaches can be applied to reroute trained-immunity-regulating drugs to myeloid (progenitor) cells to either promote trained immunity or to prevent its induction (FIG. 4). Additionally, trainedimmunity-regulating nanotherapies may display direct, pleiotropic pro- or anti-inflammatory effects.

\section{Trained immunity-promoting nanotherapeutics}

Trained immunity is characterized by quantitatively and qualitatively enhanced innate immune responses owing to metabolic and epigenetic rewiring of the myeloid cell compartment. Trained immunity can be induced to increase immunological resistance against a range of infections, including possibly against SARS-CoV-2, or to combat cancerous malignancies.

Infection and COVID-19. Several pathogens and vaccines can induce trained immunity and enhance the immune response to a subsequent encounter with an unrelated pathogen, and therefore, inducing trained immunity is a promising approach to combat infections. For example, BCG vaccination has been proposed as a strategy to reduce COVID-19-related morbidity and mortality ${ }^{115-117}$, and retrospective observational studies showed that fewer health-care workers with a history of BCG vaccination ${ }^{118}$ showed positive serology to SARS-CoV-2 (IgG), as compared to individuals without BCG vaccination. In another retrospective study, it has been shown that a recent (that is, less than 5 years old) vaccination with BCG does not correlate with hyperinflammation and is not associated with increased incidence of symptoms during the COVID-19 outbreak in the Netherlands ${ }^{119}$. Furthermore, in a double-blind randomized controlled trial, BCG vaccination led to an $80 \%$ reduction of viral respiratory infections in 200 elderly individuals ${ }^{120}$. Although this trial did not include SARS-CoV-2 infection, together these studies support the hypothesis that BCG vaccination may provide nonspecific protection against infections. Ongoing randomized controlled trials (NCT04659941, NCT04384549, NCT04537663) will shed a light on the potential of BCG vaccination in protection against infections, and vaccines eliciting trained immunity may further mitigate the impact of emerging pathogens beyond SARS-CoV-2.

Overcoming immune paralysis. In addition to infection prevention, trained-immunity-inducing agents might also be used to overcome immune paralysis, a condition that often occurs in individuals with sepsis. Immune paralysis is characterized by a functional state in which immune responses are muted as an evolutionary mechanism to prevent damage caused by long-lasting inflammatory responses ${ }^{121}$. Through similar mechanisms, immune paralysis also occurs after severe hyperinflammatory episodes as a result of cardiovascular events, such 
as stroke or myocardial infarction. Therefore, individuals suffering from immune paralysis become susceptible to (secondary) infections, such as pneumonia, which is a major cause of death in individuals hospitalized because of sepsis or a cardiovascular event ${ }^{122-124}$. $\beta$-Glucan has the potential to revert immune paralysis, as assessed by ex vivo stimulation of monocytes from intensive-care sepsis patients ${ }^{125}$. To overcome immune paralysis in vivo, sophisticated therapies must be developed to restore the balance between pro- and anti-inflammatory pathways. These therapies could, for example, be based on $\beta$-glucan-derivatives or immunoregulatory proteins such as GM-CSF and IL-7 (REF. ${ }^{122}$ ). We envision therapeutic opportunities for trained-immunity-inducing nanotherapies in individuals suffering from immune paralysis owing to sepsis, stroke, myocardial infarction or other acute inflammatory conditions that lead to immune paralysis.

Overcoming immunosuppression in cancer. Tumours escape destruction by the immune system through a process called immunoediting, which interferes with
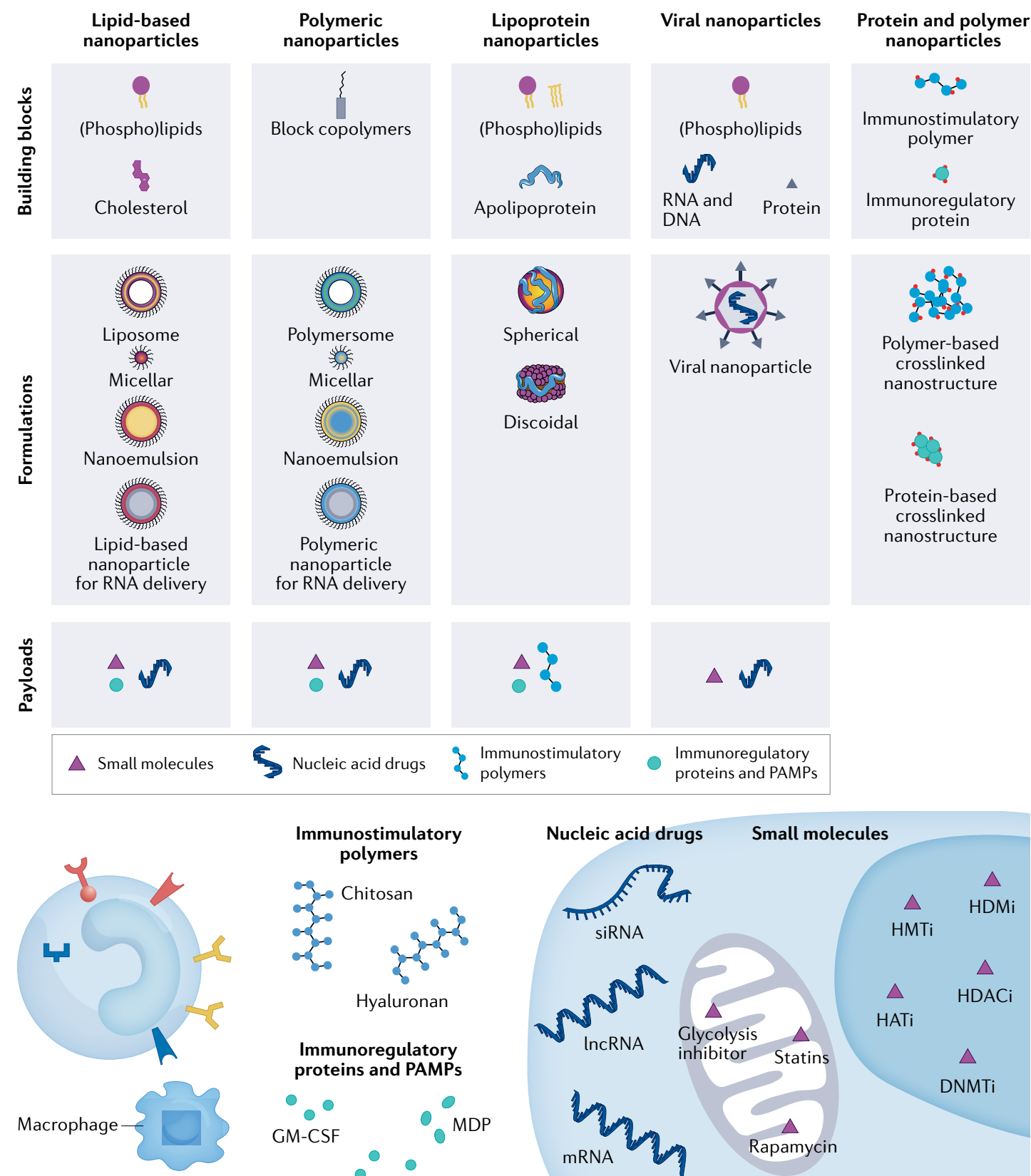

Immunostimulatory polymers

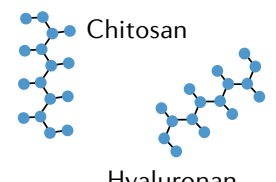

Immunoregulatory proteins and PAMPs

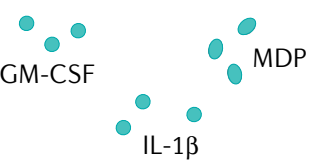

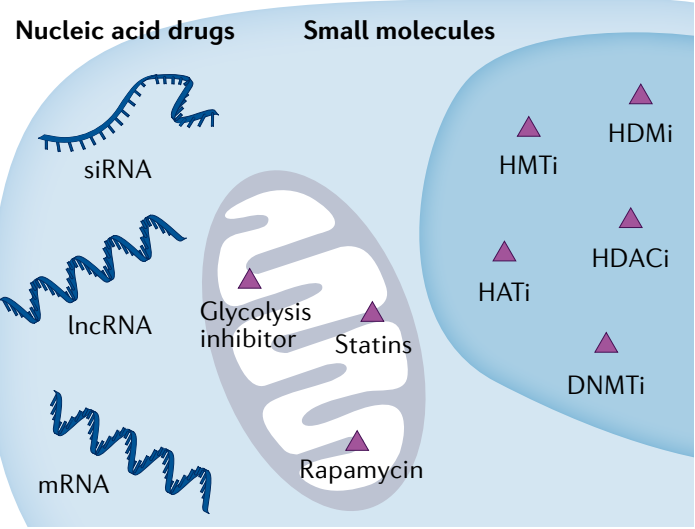

Fig. 3 | Nanoparticle platforms. An overview of nanoparticle platforms that can deliver trained-immunity-regulating payloads, including small molecules, RNA therapeutics, immunoregulatory proteins and immunostimulatory polymers. DNMTi, DNA methyltransferase inhibitor; GM-CSF, granulocyte-macrophage colony-stimulating factor; HATi, histone acetyltransferase inhibitor; $\mathrm{HDACi}$, histone deacetylase inhibitor; $\mathrm{HDMi}$, histone demethylase inhibitor; $\mathrm{HMTi}$, histone methyltransferase inhibitor; IL-1 $\beta$, interleukin-1 $\beta$; IncRNA, long non-coding RNA; MDP, muramyl dipeptide; PAMP, pathogen-associated molecular pattern; siRNA, small interfering RNA. 
Promotion of trained immunity

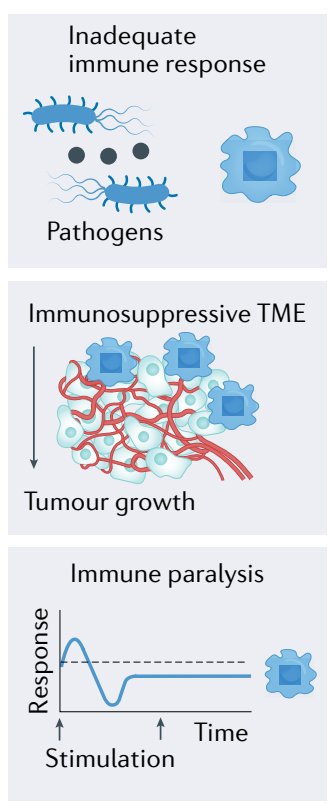

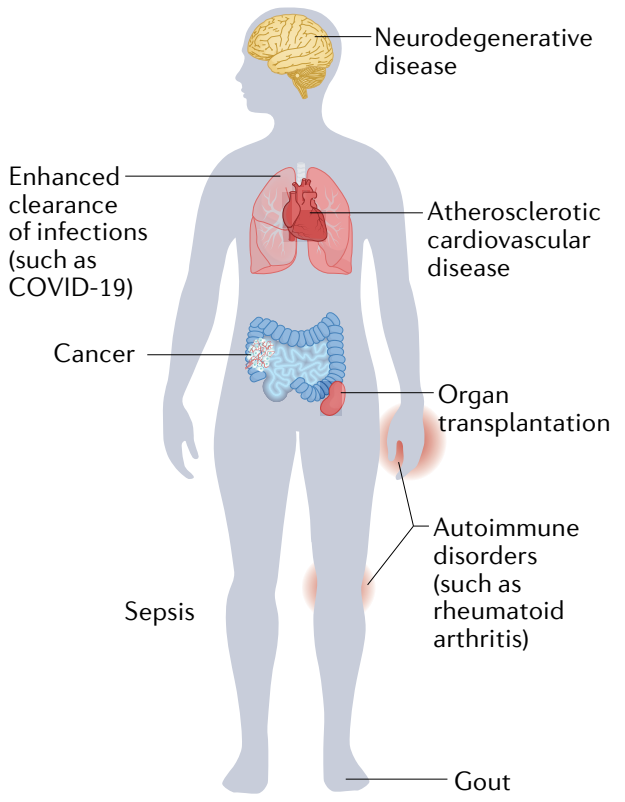

Inhibition of trained immunity

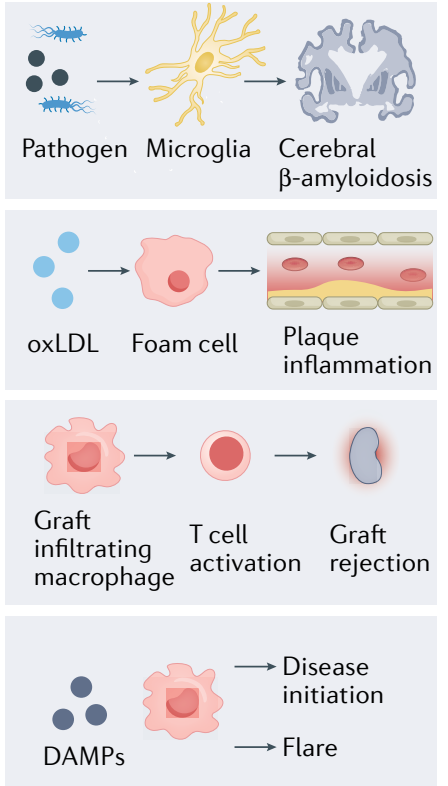

Fig. 4 | Trained-immunity-regulating nanotherapies in clinical scenarios. Trained immunity can be induced to combat cancer and increase resistance to infection, for example, against COVID-19, or trained immunity can be inhibited in conditions characterized by an exacerbated immune response. DAMP, damage-associated molecular pattern; oxLDL, oxidized low-density lipoprotein; TME, tumour microenvironment.

several aspects of normal immune activation ${ }^{126}$. First, tumour cells lose their ability to effectively present antigen (signal 1) owing to downregulation of major histocompatibility complex (MHC) molecules or presentation of mutated and thus unrecognizable antigens. Functional antigen presentation would be required to trigger anti-tumour T cell responses. Second, tumours disrupt the delicate balance of co-stimulatory and co-inhibitory signals (signal 2), further impairing $\mathrm{T}$ cell activation ${ }^{127,128}$. This loss of immunogenicity is exemplified by the upregulation of programmed cell death 1 ligand 1 (PDL1) by tumour cells. PDL1 is a co-inhibitory signal that prevents T cell-mediated tumour cell killing ${ }^{129}$. Immunotherapies, such as chimeric antigen receptor $\mathrm{T}$ cell therapy and checkpoint inhibition, focus on the adaptive arm of the immune system, targeting signals 1 and 2 . However, components of the innate immune system are also involved, generating an immunosuppressive tumour microenvironment consisting of tumour-associated myeloid cells and myeloid-derived suppressor cells, reducing the effectiveness of the abovementioned immunotherapies ${ }^{130}$. The imbalance of anti-tumour and pro-tumour myeloid cells in the tumour microenvironment stems, in part, from their aberrant production in the bone marrow ${ }^{131,132}$. In response to cytokines and growth factors produced by tumour cells ${ }^{133}$, the bone marrow produces immature cells, which are 'polarized' following their arrival in the tumour microenvironment to assist in sustaining the immunosuppressive milieu, in essence creating an inescapable positive feedback loop. This cancer-mediated immune cell modulation by HSPCs in the bone marrow may be counteracted and/or reversed by trained immunity induction. Rebalancing innate immune cell production bias from aberrant haematopoiesis to 'trained' myelopoiesis may overcome the immunosuppressive tumour microenvironment ${ }^{134}$.

The trained immunity inducer BCG is used as immunotherapy in individuals with non-muscleinvasive bladder cancer ${ }^{134}$. This intravesical treatment induces a local immune response that is based on the activation of trained immunity ${ }^{19}$. Because drugs administered to the bladder are not systemically distributed in the body, BCG, a live bacterium, can be safely applied. In addition to this local effect, a systemic effect has been reported, highlighted by the increased production of several key trained immunity cytokines by blood-derived monocytes ${ }^{135}$. To develop bone-marrow-engaging, trained-immunity-inducing nanotherapeutics that can be safely applied intravenously, we decorated nanobiologics with the smallest molecular structure of BCG capable of inducing trained immunity, that is, the NOD2-activating molecule muramyldipeptide (MDP) ${ }^{6}$. Screening of a library of nanobiologics surface-functionalized with MDP identified a formulation, named $\mathrm{MTP}_{10}-\mathrm{HDL}$ (FIG. 5a), with potent trained-immunity-inducing features (FIG. 5b) and a favourable biodistribution profile. Upon intravenous administration, $\mathrm{MTP}_{10}-\mathrm{HDL}$ immunotherapy significantly inhibited tumour growth and sensitized the resistant B16F10 melanoma tumour mouse model to checkpoint inhibition therapy (FIG. 5c). Furthermore, the anti-tumour effect of this nanobiologic immunotherapy is transferable through bone marrow transplantation into untreated mice. In-depth analysis of the bone marrow uncovered a shift in bone marrow immune cell production 
towards 'trained' myelopoiesis. The resulting increase in 'trained' monocytes and neutrophils rebalanced the tumour microenvironment, leading to tumour growth inhibition $^{6,33}$. Similarly, $\beta$-glucan has been shown to prophylactically induce trained immunity against cancer, achieving anti-tumour responses perpetuated by innate immune training through granulopoiesis and neutrophil reprogramming.

Trained immunity as an adjuvant. Trained-immunityinduced activation of the innate immune system could also enhance the efficacy of cancer vaccines. Adjuvant technology for most infection-preventing vaccines consists of potent pattern recognition receptor ligands, which are locally injected with the poorly immunogenic vaccine antigens to kick-start the immune cascade ${ }^{136}$. In contrast to classical vaccines that use foreign antigens, therapeutic cancer vaccines use self-antigen and have to overcome the immunosuppressive tumour microenvironment. The additional immunological boost provided by trained immunity induction could be advantageous in overcoming this hurdle.

Trained immunity induction as an anti-cancer strategy has been established for BCG, $\beta$-glucan and the trained immunity-inducing nanobiologic immunotherapy. Nanomedicine strategies could also be based on a clinically used oncolytic virus coding for GM-CSF. This oncolytic virotherapy, called talimogene laherparepvec (T-VEC), inhibits histone deacetylase, and has considerable off-target effects, resulting in remission of metastases, which is often contributed to immunogenic cell death. Trained immunity induction through GM-CSF signalling could be a key mechanism in the observed remission ${ }^{137}$. In addition, encapsulation of other trained immunity inducers, such as $\beta$-glucan and epigenetic drugs, could be effective.

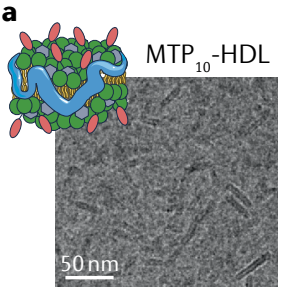

d

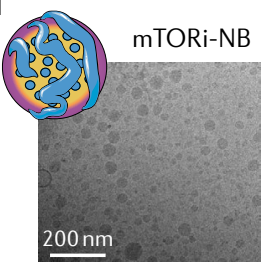

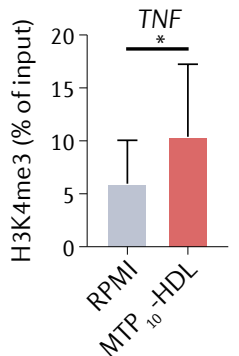

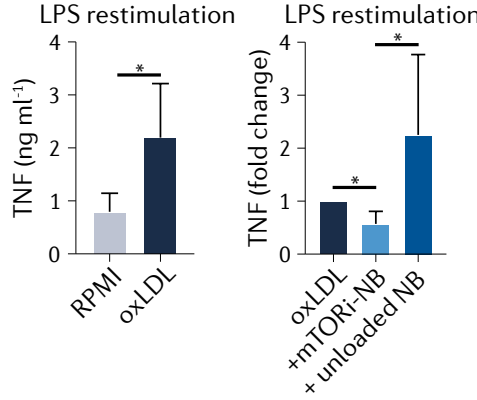

c

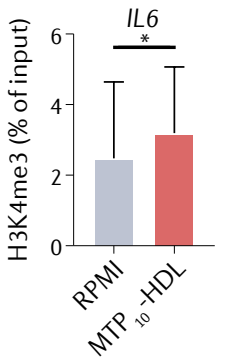

f

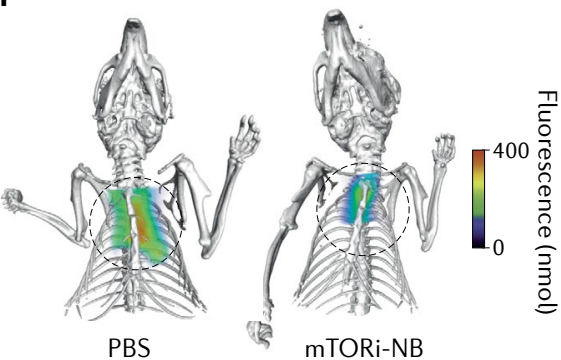

$\mathbf{g}$

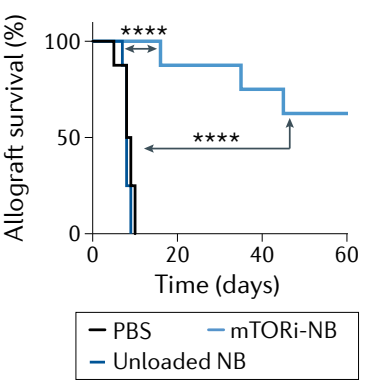

Fig. 5 | Applications of nanoimmunotherapies. a | Schematic representation (left) and cryo-transmission electron microscopy (cryo-TEM) image (right) of high-density lipoprotein (HDL)-mimicking nanobiologics (NB) loaded with 10 mol\% muramyl tripeptide $\left(\mathrm{MTP}_{10}-\mathrm{HDL}\right)^{6} . \mathbf{b} \mid$ Chromatin immunoprecipitation (ChIP)-quantitative polymerase chain reaction (qPCR) of peripheral blood mononuclear cells shows increased $\mathrm{H} 3 \mathrm{~K} 4$ methylation on the tumor necrosis factor (TNF) and interleukin-6 (IL-6) promoters after incubation with MTP $_{10}-\mathrm{HDL}$ as compared to control (Roswell Park Memorial Institute, RPMI; $n=3$ ). Error bars represent the standard deviation. $\mathbf{c}$ | Tumour growth curve of B16F10 melanoma in C57Bl/ 6 mice. Mice were inoculated with $10^{5}$ B16F10 cells at day -7 and MTP $_{10}-\mathrm{HDL}\left(\mathrm{MTP}\right.$ at $1.5 \mathrm{mg} \mathrm{kg}^{-1}$ ) was intravenously administered at days 0,2 and 4 . In one group, MTP ${ }_{10}-\mathrm{HDL}$ treatment was continued; mice were also injected on days 6 and 8 . Checkpoint inhibitors anti-cytotoxic T lymphocyte antigen 4 (anti-CTLA4) and anti-programmed cell death 1 (anti-PD1) (both $200 \mu \mathrm{g}$ per mouse) were administered intraperitoneally twice per week, starting at day 2 . The primary outcome was the comparison between checkpoint inhibitor monotherapy versus combination with MTP ${ }_{10}-\mathrm{HDL}$. Significance was calculated for tumour growth rate (black) and tumour size (green). Data are represented at mean \pm standard error of the mean. $\mathbf{d}$ |Schematic representation and cryo-TEM image of HDL-mimicking NB loaded with the mechanistic target of rapamycin (mTOR) inhibitor (mTORi-NB). e | Human primary monocytes were incubated with oxidized LDL (oxLDL) for 24 hours, and after a 5-day rest, cells were restimulated with lipopolysaccharide (LPS). oxLDL amplifies the TNF production upon LPS stimulation, as measured by enzyme-linked immunosorbent assay (ELISA). When human monocytes are incubated with oxLDL in combination with mTORi-NB, TNF production is reduced, as compared to unloaded NB or oxLDL only $(n=6)$. $\mathbf{f} \mid$ Atherosclerosis-prone $A_{p o e^{-/-}}$mice were held on a Western diet for 12 weeks to develop advanced plaques. The mice received four intravenous injections of mTORi-NB (mTORi at $5 \mathrm{mg} \mathrm{kg}^{-1}$ ) or phosphate buffered saline (PBS) for one week, before being subjected to fluorescence molecular tomography (FMT) imaging ${ }^{177}$. mTORi-NB-treated mice showed less fluorescence in the aortic root area, indicative of reduced protease activity - a measure of macrophage inflammation. $\mathbf{g}$ | Assessment of heart allograft survival in C57Bl/6 mice treated with mTORi-NBs $\left(\mathrm{mTORi}\right.$ at $\left.5 \mathrm{mg} \mathrm{kg}^{-1}\right)$. The mice received unloaded $35 \mathrm{~nm}$ NB or PBS directly before as well as 2 days and 5 days posttransplantation, $n=8$ per group. Allograft survival was monitored by micro-ultrasound imaging. $P$ values were calculated using a log-rank test. ${ }^{\star} P<0.05,{ }^{\star \star} P<0.01,{ }^{* \star \star} P<0.001,{ }^{* \star \star \star} P<0.0001$. Panels a-c adapted with permission from REF. ${ }^{6}$, Elsevier. Panels $\mathbf{d}$ and $\mathbf{g}$ adapted with permission from REF. ${ }^{62}$, AAAS. Panels $\mathbf{e}$ and $\mathbf{f}$ adapted with permission from REF. ${ }^{140}$, AAAS. 


\section{Trained immunity-inhibiting nanotherapeutics}

Many immune-mediated conditions could be combatted through the prevention or reversal of 'dysfunctional' trained immunity.

Inflammatory disease. Maladaptive trained immunity mechanisms have been reported in atherosclerosis, a chronic inflammatory disease, which is the main underlying cause of cardiovascular pathologies. Non-microbial stimuli relevant to atherosclerosis, such as oxLDL, induce training in human monocytes, reflected in the increased production of pro-inflammatory cytokines and underlying epigenetic changes ${ }^{48}$. Similar monocyte phenotypes have been detected in individuals with coronary artery disease ${ }^{138}$ or dyslipidaemia ${ }^{139}$. Prevention of oxLDL-induced trained immunity can be achieved in vitro through mTOR inhibition with rapamycin. Similarly, nanobiologics containing the mTOR inhibitor (mTORi-NB) prevent oxLDLinduced pro-inflammatory cytokine production in human monocytes in vitro ${ }^{140}$ (FIG. $5 \mathrm{~d}$,e). Importantly, in atherosclerosis-prone $A p o e^{-/-}$mice that were fed a Western diet for 12 weeks, a one-week mTORi-NB treatment regimen reduced plaque inflammation (FIG. 5f). Such highly effective, anti-inflammatory nanotherapeutics could also be applied in an acute cardiovascular event, such as myocardial infarction, or to treat high-risk patients with elevated biomarkers indicative of systemic inflammation ${ }^{141}$.

In addition to atherosclerosis, trained immunity plays a part in neurodegenerative diseases. For example, peripheral inflammatory stimuli can induce training or tolerance in brain-resident macrophages, also known as microglia, through epigenetic reprogramming ${ }^{142}$. Here, training increases $\beta$-amyloidosis and inflammation, whereas tolerized microglia have the opposite effect. Interestingly, tolerized microglia also reduce neuronal damage after stroke. Given that HSPCs in the bone marrow have a key role in the regulation of trained immunity, the blood-brain barrier does not pose any restrictions for this therapeutic approach. Gout, a form of inflammatory arthritis, is caused by the deposition of urate crystals in joints. These urate crystals as well as soluble urate can cause epigenetic and translational reprogramming of monocytes, resulting in increased IL- $1 \beta$ production $^{50,143}$, indicating a contribution of trained immunity to this disease. The inhibition of mTOR signalling may be a promising approach to inhibit trained immunity, because mTOR activation is involved in both pathways ${ }^{50}$.

Autoimmune disorders. Many autoimmune and autoinflammatory diseases, such as rheumatoid arthritis, systemic lupus erythematosus, systemic sclerosis, sarcoidosis, diabetes mellitus type 1 , and familial Mediterranean fever, display features of a trained immunity phenotype ${ }^{144}$. For example, changes in cellular metabolism and/or epigenetic rewiring underlie the increased cytokine production of innate immune cells in these diseases. Trained immunity could therefore have a crucial role in the initiation of these diseases, but also in the persistence or aggravation of symptoms. Nanotherapies could be developed for specific trained immunity pathways in these autoimmune diseases. For example, a nanotherapeutic treatment has been tested for experimental autoimmune encephalitis, a mouse model of multiple sclerosis ${ }^{145}$. Here, lipid nanoparticle formulations have been designed that contain modified autoantigen-encoding mRNA, which is taken up by $\mathrm{CD} 11 \mathrm{c}^{+}$cells in the spleen, resulting in antigen-specific tolerance. Although it is different to antigen-unspecific trained immunity regulation, nanoparticle-innate immune cell interaction holds great promise for the treatment of autoimmune diseases.

Organ transplantation. The ultimate goal in transplantation medicine is optimal graft function and the prevention of allograft rejection through the induction of immunological tolerance. The current standard of care is chronic immunosuppression, which is associated with major side effects, such as increased cancer incidence and susceptibility to infection. Allograft rejection is driven by the adaptive immune system. However, the onset of allograft rejection is triggered by innate immune cell activation ${ }^{146}$. Therefore, induction of an immune memory could be prevented by targeting myeloid cell inflammation. Moreover, allograft transplantation induces trained innate immunity through vimentin and HMGB1 signalling ${ }^{7}$, which can be inhibited by myeloid-cell-avid mTORi-NBs. Indeed, a brief mTORi-NB treatment regimen consisting of three intravenous injections significantly increased allograft survival without the need for chronic immunosuppression in a mouse heart transplantation model ${ }^{7,62}$ (FIG. 5g). Mechanistically, mTORi-NB treatment rebalances the immune system and prevents the induction of trained immunity, resulting in expansion of $\mathrm{CD}^{+}$regulatory $\mathrm{T}\left(\mathrm{T}_{\text {reg }}\right)$ cells at the expense of $\mathrm{CD}^{+}$cytotoxic $\mathrm{T}$ cells, subsequently delaying or completely preventing allograft rejection.

Hyperinflammatory episodes. The initiation and resolution of inflammation are complex mechanisms, which must be precisely calibrated to achieve the best possible clinical outcome ${ }^{147}$. For example, excessive inflammation after myocardial infarction can cause local tissue damage and worsen cardiac outcome ${ }^{148-150}$. Importantly, a hyperinflammatory episode can have major systemic consequences through the induction of trained immunity-like programs. Similar harmful cascades can also occur following infections. For example, an epidemic of cardiovascular events follows the normal influenza season, most probably caused by infection-induced rewiring of immune programs, which exacerbate ongoing cardiovascular inflammation, thereby increasing the likelihood of atherothrombotic events ${ }^{151}$. Individuals suffering from a hyperinflammatory episode, following cardiovascular events or infection, could benefit from anti-trained-immunity (nano)therapy that prevents immunological rewiring and circumvents detrimental downstream consequences. Although more research is needed to prove this hypothesis, an anti-inflammatory therapy with canakinumab, a therapeutic monoclonal antibody targeting IL- $1 \beta$, has already been shown to reduce the recurrence rate of cardiovascular events in a clinical trial ${ }^{152}$. 


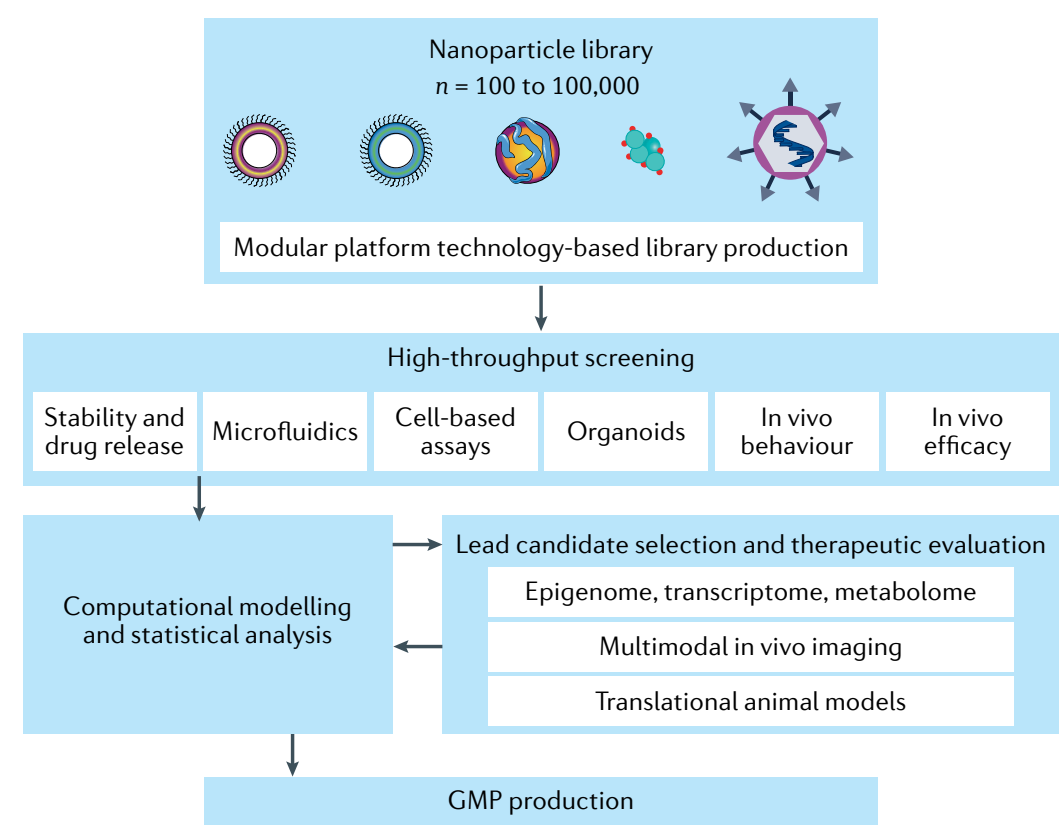

Fig. 6 | Translation from preclinical concept to clinical trials. Modular platform technology facilitates the seamless incorporation of drugs into a particular platform. High-throughput screening of nanomaterial libraries includes in vitro systems and in vivo assays. Lead candidate selection is supported by computational modelling, and therapeutic evaluation of the lead candidate includes omics approaches, multimodal in vivo imaging and the use of translational animal models. This approach will generate large data sets from which meaningful information can be extracted using non-biased computational modelling methods, before translational steps to good manufacturing practice (GMP) production can be made. these discrepancies between in vitro and in vivo nanoparticle behaviour, we advocate a two-pronged approach that involves the systematic screening of large libraries of nanomaterials, concurrently in vitro and in mouse models in vivo (FIG. 6). Thus, from a single platform, libraries of differently composed, sized, shaped or charged nanoparticles could be established ${ }^{163}$. Nanoparticles in the library could be individually labelled, for example, by radiolabelling their therapeutic payloads or molecular building blocks, allowing quantitative detection by nuclear imaging techniques ${ }^{164}$. Similarly, fluorophores or stable isotopes could be integrated to study nanoparticle behaviour using optical techniques (NIRF imaging ${ }^{165}$, intravital microscopy ${ }^{166}$, immunofluorescence ${ }^{167}$ and flow cytometry ${ }^{6}$ ) and methods based on mass spectrometry, such as liquid chromatography-mass spectrometry or mass cytometry ${ }^{168,169}$ (BOX 2). Additionally, non-invasive imaging could provide dynamic and longitudinal biodistribution information at the whole-organism level. Finally, multi-omics approaches, including chromatin immunoprecipitation followed by sequencing (ChIP-seq) and assay for transposase-accessible chromatin using sequencing (ATAC-seq) (epigenome), RNA-sequencing (transcriptome) and cytometry methods (immune landscape) should be applied to understand the interaction of nanomaterials with the immune system, from the molecular to the cellular level and beyond ${ }^{170}$. These studies will generate large data sets from which meaningful information can be extracted using non-biased computational modelling methods, including supervised and unsupervised machine learning ${ }^{171}$.

An additional level of refinement can be achieved by a concept called modularity of functionalization, that is, designing nanoparticle platforms that allow straightforward integration of therapeutic functionalities, without compromising the platform's in vivo features. Modularity is an innate feature of RNA-based therapeutics ${ }^{172}$, but can also be implemented for other types of therapeutics, for example, smart pro-drug approaches for small-molecule drugs $^{62}$. Regulatory bodies, such as the US Food and Drug Administration (FDA) and European Medicines Agency (EMA), should develop an approval process for nanomedicine platforms that can be modularly functionalized with diverse therapeutic payloads. Indeed, one reason that the COVID-19 mRNA vaccines were rapidly developed and deemed effective and safe is because the nanoparticle carrier system had previously been extensively evaluated, albeit carrying different nucleic acids ${ }^{173}$.

As new pathways and mechanisms are being unravelled, an increasingly defined picture of trained immunity is emerging ${ }^{3}$. The therapeutic regulation of trained immunity requires innovative therapeutics with the ability to change the functional programs of HSPCs (in the bone marrow and spleen). Nanomedicine has the potential to develop valuable trained-immunity-regulating methods, and the merging of nanomedicine, immunology, immunotherapy, in vivo imaging and artificial intelligence will yield nanoparticle-based immunotherapies that should revolutionize the treatment of immune-mediated diseases.

Published online 7 February 2022 
1. Irvine, D. J. \& Dane, E. L. Enhancing cancer immunotherapy with nanomedicine Nat. Rev. Immunol. 20, 321-334 (2020)

2. Netea, M. G. et al. Trained immunity: a program of innate immune memory in health and disease. Science 352, 427 (2016).

This review discusses the discovery of trained immunity.

3. Netea, M. G. et al. Defining trained immunity and its role in health and disease. Nat. Rev. Immunol. 20, 375-388 (2020).

4. Cheng, S.-C. et al. mTOR- and HIF-1 $\alpha$-mediated aerobic glycolysis as metabolic basis for trained immunity. Science 345, 1250684 (2014).

5. Kleinnijenhuis, J. et al. Bacille Calmette-Guérin induces NOD2-dependent nonspecific protection from reinfection via epigenetic reprogramming of monocytes. Proc. Natl Acad. Sci. USA 109, 17537-17542 (2012).

6. Priem, B. et al. Trained immunity-promoting nanobiologic therapy suppresses tumor growth and potentiates checkpoint inhibition. Cell 183, 786-801 (2020).

In this proof-of-principle study, trained immunity-inducing nanobiologics are used to combat tumour growth.

7. Braza, M. S. et al. Inhibiting inflammation with myeloid cell-specific nanobiologics promotes organ transplant acceptance. Immunity 49, 819-828.e6 (2018).

This study demonstrates the application of trained immunity-inhibiting nanobiologics to induce tolerance after heart allograft transplantation.

8. Levine, D. B. The hospital for the ruptured and crippled: William Bradley Coley, Third Surgeon-in-Chief 1925-1933. HSS J. 4, 1-9 (2008).

9. Coley, W. B. II Contribution to the knowledge of sarcoma. Ann. Surg. 14, 199-220 (1891).

10. Coley, W. B. The treatment of inoperable sarcoma with the 'mixed toxins of erysipelas and Bacillus prodigiosus. J. Am. Med. Assoc. 31, 456-465 (1898).

11. Morales, A., Eidinger, D. \& Bruce, A. W. Intracavitary Bacillus Calmette-Guérin in the treatment of superficial bladder tumors. J. Urol. 116, 180-182 (1976).

12. Mitroulis, I. et al. Modulation of myelopoiesis progenitors is an integral component of trained immunity. Cell 172, 147-161.e12 (2018). This study shows that trained immunity modulates haematopoietic progenitors in the bone marrow.

13. Mulder, W. J. M., Ochando, J., Joosten, L. A. B. Fayad, Z. A. \& Netea, M. G. Therapeutic targeting of trained immunity. Nat. Rev. Drug Discov. 18 553-566 (2019).

14. Moorlag, S. J. C. F. M. et al. $\beta$-glucan induces protective trained immunity against Mycobacterium tuberculosis infection: a key role for IL-1. Cell Rep. 31 , 107634 (2020)

15. Kulkarni, J. A., Cullis, P. R. \& van der Meel, R. Lipid nanoparticles enabling gene therapies: from concepts to clinical utility. Nucleic Acid Ther. 28, 146-157 (2018).

16. Fredman, G. et al. Targeted nanoparticles containing the proresolving peptide Ac2-26 protect against advanced atherosclerosis in hypercholesterolemic mice. Sci. Transl Med. 7, 275 ra20 (2015).

17. Janeway, C. A. \& Medzhitov, R. Innate immune recognition. Annu. Rev. Immunol. 20, 197-216 (2002).

18. Rock, K. L., Lai, J.-J. \& Kono, H. Innate and adaptive immune responses to cell death. Immunol. Rev. 243 191-205 (2011).

19. Bretscher, P. \& Cohn, M. A theory of self-nonself discrimination: paralysis and induction involve the recognition of one and two determinants on an antigen, respectively. Science 169, 1042-1049 (1970).

20. Schnare, M. et al. Toll-like receptors control activation of adaptive immune responses. Nat. Immunol. $\mathbf{2}$, 947-950 (2001)

21. Pasare, C. \& Medzhitov, R. Toll-dependent control mechanisms of CD4 T cell activation. Immunity 21, 733-741 (2004)

22. Curtsinger, J. M. et al. Inflammatory cytokines provide a third signal for activation of naive $\mathrm{CD} 4^{+}$and $\mathrm{CD} 8^{+}$ T cells. J. Immunol. 162, 3256-3262 (1999).

23. Morrison, S. J. \& Scadden, D. T. The bone marrow niche for haematopoietic stem cells. Nature $\mathbf{5 0 5}$, 327-334 (2014).

24. Seita, J. \& Weissman, I. L. Hematopoietic stem cell: self-renewal versus differentiation. Wiley Interdiscip. Rev. Syst. Biol. Med. 2, 640-653 (2010).
25. Quintin, J. et al. Candida albicans infection affords protection against reinfection via functional reprogramming of monocytes. Cell Host Microbe 12, 223-232 (2012)

26. Kleinnijenhuis, J. et al. BCG-induced trained immunity in NK cells: role for non-specific protection to infection Clin. Immunol 155, 213-219 (2014).

27. Moorlag, S. J. C. F. M. et al. BCG vaccination induces long-term functional reprogramming of human neutrophils. Cell Rep. 33, 108387 (2020)

28. Naik, S. et al. Inflammatory memory sensitizes skin epithelial stem cells to tissue damage. Nature $\mathbf{5 5 0}$, 475-480 (2017)

29. Cirovic, B. et al. BCG vaccination in humans elicits trained immunity via the hematopoietic progenitor compartment. Cell Host Microbe 28, 322-334.e5 (2020).

30. Saeed, S. et al. Epigenetic programming of monocyteto-macrophage differentiation and trained innate immunity. Science 345, 1251086 (2014).

31. Donahue, N. D., Acar, H. \& Wilhelm, S. Concepts of nanoparticle cellular uptake, intracellular trafficking, and kinetics in nanomedicine. Adv. Drug Deliv. Rev. 143, 68-96 (2019).

32. Kaufmann, E. et al. BCG educates hematopoietic stem cells to generate protective innate immunity against tuberculosis. Cell 172, 176-190 e19 (2018).

33. Kalafati, L. et al. Innate immune training of granulopoiesis promotes anti-tumor activity. Cell 183, 771-785.e12 (2020).

34. Hole, C. R. et al. Induction of memory-like dendritic cell responses in vivo. Nat. Commun. 10, 2955 (2019).

35. Durai, V. $\&$ Murphy, K. M. Functions of murine dendritic cells. Immunity 45, 719-736 (2016)

36. Cooper, M. A. et al. Cytokine-induced memory-like natural killer cells. Proc. Natl Acad. Sci. USA 106 1915-1919 (2009).

37. Romee, R. et al. Cytokine activation induces human memory-like NK cells. Blood 120, 4751-4760 (2012).

38. Romee, R. et al. Cytokine-induced memory-like natural killer cells exhibit enhanced responses against myeloid leukemia. Sci. Transl Med. 8, 357 ra1 23 (2016).

39. Weizman, O.-E. et al. Mouse cytomegalovirusexperienced ILC $1 \mathrm{~s}$ acquire a memory response dependent on the viral glycoprotein m12. Nat. Immunol. 20, 1004-1011 (2019).

40. Walk, J. et al. Outcomes of controlled human malaria infection after BCG vaccination. Nat. Commun. 10, 874 (2019).

41. Brown, G. D. \& Gordon, S. A new receptor for $\beta$-glucans. Nature 413, 36-37 (2001).

42. Tang, C. et al. Inhibition of dectin-1 signaling ameliorates colitis by inducing Lactobacillus-mediated regulatory $\mathrm{T}$ cell expansion in the intestine. Cell Host Microbe 18, 183-197 (2015).

43. Eramo, M. J. \& Mitchell, C. A. Regulation of Ptdlns $(3,4,5) \mathrm{P}_{3} /$ Akt signalling by inositol polyphosphate 5-phosphatases. Biochem. Soc. Trans. 44, 240-252 (2016)

44. Saz-Leal, P. et al. Targeting SHIP-1 in myeloid cells enhances trained immunity and boosts response to infection. Cell Rep. 25, 1118-1126 (2018).

45. O'Connell, R. M., Chaudhuri, A. A., Rao, D. S. \& Baltimore, D. Inositol phosphatase SHIP1 is a primary target of miR-155. Proc. Natl Acad. Sci. USA 106 7113-7118 (2009).

46. Babar, I. A. et al. Nanoparticle-based therapy in an in vivo microRNA-155 (miR-155)-dependent mouse model of lymphoma. Proc. Natl Acad. Sci. USA 109 E1695-E1704 (2012).

47. Caruso, R., Warner, N., Inohara, N. \& Núñez, C. NOD 1 and NOD2: signaling, host defense, and inflammatory disease. Immunity 41, 898-908 (2014)

48. Bekkering, S. et al. Oxidized low-density lipoprotein induces long-term proinflammatory cytokine production and foam cell formation via epigenetic reprogramming of monocytes. Arterioscler. Thromb. Vasc. Biol. 34, 1731-1738 (2014)

49. van der Valk, F. M. et al. Oxidized phospholipids on lipoprotein(a) elicit arterial wall inflammation and an inflammatory monocyte response in humans. Circulation 134, 611-624 (2016).

50. Cabău, G., Crișan, T. O., Klück, V., Popp, R. A. \& Joosten, L. A. B. Urate-induced immune programming consequences for gouty arthritis and hyperuricemia. Immunol. Rev. 294, 92-105 (2020).

51. Miller, Y. I. et al. Oxidation-specific epitopes are danger-associated molecular patterns recognized by pattern recognition receptors of innate immunity Circ. Res. 108, 235-248 (2011).
52. Matsunaga, N., Tsuchimori, N., Matsumoto, T. \& li, M TAK-242 (resatorvid), a small-molecule inhibitor of Toll-Like Receptor (TLR) 4 signaling, binds selectively to TLR4 and interferes with Interactions between TLR4 and its adaptor molecules. Mol. Pharmacol. 79 34-41 (2011).

53. Schnack, L. et al. Mechanisms of trained innate immunity in oxldl primed human coronary smooth muscle cells. Front. Immunol. 10, 13 (2019).

54. Bekkering, S. et al. Metabolic induction of trained immunity through the mevalonate pathway. Cell 172 135-143.e9 (2018).

55. Arts, R. J. W. et al. Immunometabolic pathways in BCG-induced trained immunity. Cell Rep. 17, 2562-2571 (2016).

56. Mulder, W. J. M. et al. High-density lipoprotein nanobiologics for precision medicine. Acc. Chem. Res. 51, 127-137 (2018)

57. Tang, J. et al. Inhibiting macrophage proliferation suppresses atherosclerotic plaque inflammation. Sci. Adv. 1, e1400223 (2015)

58. Duivenvoorden, $\mathrm{R}$, et al. A statin-loaded reconstituted high-density lipoprotein nanoparticle inhibits atherosclerotic plaque inflammation. Nat. Commun. 5 3065 (2014).

59. Binderup, T. et al. Imaging-assisted nanoimmunotherapy for atherosclerosis in multiple species. Sci. Transl Med. 11, eaaw7736 (2019).

60. Arts, R. J. W. et al. Glutaminolysis and fumarate accumulation integrate immunometabolic and epigenetic programs in trained immunity. Cell Metab. 24, 807-819 (2016).

61. Mills, E. L. et al. Succinate dehydrogenase supports metabolic repurposing of mitochondria to drive inflammatory macrophages. Cell 167, 457-470.e13 (2016).

62. van Leent, M. M. T. et al. A modular approach toward producing nanotherapeutics targeting the innate immune system. Sci. Adv. 7, eabe7853 (2021).

63. Jones, P. A., Issa, J. P. J. \& Baylin, S. Targeting the cancer epigenome for therapy. Nat. Rev. Genet. 17 630-641 (2016)

64. van der Heijden, C. D. C. C. et al. Epigenetics and trained immunity. Antioxid. Redox Signal. 29, 1023-1040 (2018).

65. Naz, A., Cui, Y., Collins, C. J., Thompson, D. H. \& Irudayaraj, J. PLGA-PEG nano-delivery system for epigenetic therapy. Biomed. Pharmacother. 90 , 586-597 (2017)

66. Fanucchi, S. et al. Immune genes are primed for robust transcription by proximal long noncoding RNAs located in nuclear compartments. Nat. Genet. 51, 138-150 (2019).

67. Jin, F. et al. A high-resolution map of the three-dimensional chromatin interactome in human cells. Nature 503, 290-294 (2013).

68. Allen, T. M., Hansen, C. \& Rutledge, J. Liposomes with prolonged circulation times: factors affecting uptake by reticuloendothelial and other tissues. Biochim. Biophys. Acta 981, 27-35 (1989).

69. Krohn-Grimberghe, M. et al.

Nanoparticle-encapsulated siRNAs for gene silencing in the haematopoietic stem-cell niche. Nat. Biomed. Eng. 4, 1076-1089 (2020).

70. Hak, S., Garaiova, Z., Olsen, L. T., Nilsen, A. M $\Sigma$ de Lange Davies, $C$. The effects of oil-in-water nanoemulsion polyethylene glycol surface density on intracellular stability, pharmacokinetics, and biodistribution in tumor bearing mice. Pharm. Res. 32, 1475-1485 (2015).

71. Hong, R. L. et al. Direct comparison of liposomal doxorubicin with or without polyethylene glycol coating in C-26 tumor-bearing mice: is surface coating with polyethylene glycol beneficial? Clin. Cancer Res. 5, 3645-3652 (1999)

72. Vittaz, M. et al. Effect of PEO surface density on long-circulating PLA-PEO nanoparticles which are very low complement activators. Biomaterials 17 1575-1581 (1996).

73. Lo, C. K., Xiao, D. \& Choi, M. M. F. Homocysteine protected gold-coated magnetic nanoparticles: synthesis and characterisation. J. Mater. Chem. 17 2418 (2007).

74. Jain, T. K., Morales, M. A., Sahoo, S. K. Leslie-Pelecky, D. L. \& Labhasetwar, V. Iron oxide nanoparticles for sustained delivery of anticancer agents. Mol. Pharm. 2, 194-205 (2005).

75. Correa-Duarte, M. A., Giersig, M. ¿ Liz-Marzán, L. M. Stabilization of CdS semiconductor nanoparticles against photodegradation by a silica coating procedure. Chem. Phys. Lett. 286, 497-50 (1998). 
76. Alexis, F., Pridgen, E., Molnar, L. K. \& Farokhzad, O. C. Factors affecting the clearance and biodistribution of polymeric nanoparticles. Mol. Pharm. 5, 505-515 (2008).

77. Mitchell, M. J. et al. Engineering precision nanoparticles for drug delivery. Nat. Rev. Drug Discov. 20, 101-124 (2021).

This review discusses advances in particle design for precision medicine.

78. Matsumura, Y. \& Maeda, H. A new concept for macromolecular therapeutics in cancer chemotherapy: mechanism of tumoritropic accumulation of proteins and the antitumor agent smancs. Cancer Res. 46, 63863-92 (1986).

79. Choi, H. S. et al. Renal clearance of quantum dots. Nat. Biotechnol. 25, 1165-1170 (2007).

80. Choi, H. S. et al. Design considerations for tumour-targeted nanoparticles. Nat. Nanotechnol. 5, 42-47 (2010).

81. Tsoi, K. M. et al. Mechanism of hard-nanomaterial clearance by the liver. Nat. Mater. 15, 1212-1221 (2016).

82. Hoshyar, N., Gray, S., Han, H. \& Bao, G. The effect of nanoparticle size on in vivo pharmacokinetics and cellular interaction. Nanomedicine 11, 673-692 (2016).

83. Blanco, E., Shen, H. \& Ferrari, M. Principles of nanoparticle design for overcoming biological barriers to drug delivery. Nat. Biotechnol. 33, 941-951 (2015).

84. Torchilin, V. P. Multifunctional, stimuli-sensitive nanoparticulate systems for drug delivery. Nat. Rev. Drug Discov. 13, 813-827 (2014).

85. Allen, T. M., Hansen, C., Martin, F., Redemann, C \& Yau-Young, A. Liposomes containing synthetic lipid derivatives of poly(ethylene glycol) show prolonged circulation half-lives in vivo. Biochim. Biophys. Acta 1066, 29-36 (1991).

86. Sosnovik, D. E. et al. Fluorescence tomography and magnetic resonance imaging of myocardial macrophage infiltration in infarcted myocardium in vivo. Circulation 115, 1384-1391 (2007).

87. Frias, J. C., Williams, K. J., Fisher, E. A. \& Fayad, Z. A. Recombinant HDL-like nanoparticles: a specific contrast agent for MRI of atherosclerotic plaques. J. Am. Chem. Soc. 126, 16316-16317 (2004).

88. Metselaar, J. M., Wauben, M. H. M. Wagenaar-Hilbers, J. P. A., Boerman, O. C. \& Storm, G. Complete remission of experimental arthritis by joint targeting of glucocorticoids with long-circulating liposomes. Arthritis Rheum. 48, 2059-2066 (2003).

89. Swirski, F. K. et al. Identification of splenic reservoir monocytes and their deployment to inflammatory sites. Science 325, 612-616 (2009).

90. Leuschner, F. et al. Therapeutic siRNA silencing in inflammatory monocytes in mice. Nat. Biotechnol. 29 , 1005-1010 (2011).

91. Mayer, L. D. et al. Characterization of liposomal systems containing doxorubicin entrapped in response to $\mathrm{pH}$ gradients. Biochim. Biophys. Acta 1025 , 143-151 (1990)

92. Akinc, A. et al. The Onpattro story and the clinical translation of nanomedicines containing nucleic acid-based drugs. Nat. Nanotechnol. 14, 1084-1087 (2019).

93. [No authors listed.] Messengers of hope. Nat. Biotechnol. 39, 1 (2021).

94. Polack, F. P. et al. Safety and efficacy of the BNT162b2 mRNA Covid-19 vaccine. N. Engl. J. Med. 383, 2603-2615 (2020)

95. Baden, L. R. et al. Efficacy and safety of the mRNA1273 SARS-CoV-2 vaccine. N. Engl. J. Med. 384 403-416 (2021)

96. Rideau, E., Dimova, R., Schwille, P., Wurm, F. R $\&$ Landfester, K. Liposomes and polymersomes: a comparative review towards cell mimicking. Chem. Soc. Rev. 47, 8572-8610 (2018).

97. Kamaly, N., Xiao, Z., Valencia, P. M. Radovic-Moreno, A. F. \& Farokhzad, O. C. Targeted polymeric therapeutic nanoparticles: design, development and clinical translation. Chem. Soc. Rev. 41, 2971 (2012).

98. Estabrook, D. A., Ennis, A. F., Day, R. A \& Sletten, E. M. Controlling nanoemulsion surface chemistry with poly(2-oxazoline) amphiphiles. Chem. Sci. 10, 3994-4003 (2019).

99. Pardi, N., Hogan, M. J., Porter, F. W. \& Weissman, D. mRNA vaccines-a new era in vaccinology. Nat. Rev. Drug Discov. 17, 261-279 (2018).

100. Yildiz, I., Shukla, S. \& Steinmetz, N. F. Applications of viral nanoparticles in medicine. Curr. Opin. Biotechnol. 22, 901-908 (2011).
101. Chung, Y. H., Cai, H. \& Steinmetz, N. F. Viral nanoparticles for drug delivery, imaging, immunotherapy, and theranostic applications. Adv. Drug Deliv. Rev. 156, 214-235 (2020).

102. Tian, J.-H. et al. SARS-CoV-2 spike glycoprotein vaccine candidate NVX-CoV2373 immunogenicity in baboons and protection in mice. Nat. Commun. 12 372 (2021).

103. Marcandalli, J. et al. Induction of potent neutralizing antibody responses by a designed protein nanoparticle vaccine for respiratory syncytial virus. Cell 176 1420-1431.e17 (2019).

104. Maugeri, M. et al. Linkage between endosomal escape of LNP-mRNA and loading into EVs for transport to other cells. Nat. Commun. 10, 4333 (2019).

105. Torchilin, V. P. Recent advances with liposomes as pharmaceutical carriers. Nat. Rev. Drug Discov. 4 145-160 (2005). This comprehensive review discusses liposomes.

106. Hoseini, M. H. M., Sadeghi, S., Azizi, M. \& Pouriran, R. in Handbook of Chitin and Chitosan (eds Gopi, S., Thomas, S. \& Pius, A.) 609-639 (Elsevier, 2020).

107. Girardin, S. E. et al. Nod2 is a general sensor of peptidoglycan through muramyl dipeptide (MDP) detection. J. Biol. Chem. 278, 8869-8872 (2003)

108. Palma, A. S. et al. Ligands for the $\beta$-glucan receptor dectin-1, assigned using "designer" microarrays of oligosaccharide probes (neoglycolipids) generated from glucan polysaccharides. J. Biol. Chem. 281, 5771-5779 (2006).

109. Kamaly, N. et al. Targeted Interleukin-10 nanotherapeutics developed with a microfluidic chip enhance resolution of inflammation in advanced atherosclerosis. ACS Nano 10, 5280-5292 (2016).

110. Verbeke, R., Lentacker, I., De Smedt, S. C. \& Dewitte, $\mathrm{H}$. The dawn of mRNA vaccines: the COVID-19 case. J. Control. Release 333, 511-520 (2021).

111. Hou, X., Zaks, T., Langer, R. \& Dong, Y. Lipid nanoparticles for mRNA delivery. Nat. Rev. Mater. 6 , 1078-1094 (2021).

This review discusses lipid nanoparticles for mRNA delivery, focusing on particle design, administration routes and clinical translation.

112. Voysey, M. et al. Safety and efficacy of the ChAdOx1 nCoV-19 vaccine (AZD 1222) against SARS-CoV-2: an interim analysis of four randomised controlled trials in Brazil, South Africa, and the UK. Lancet 397, 99-111 (2021).

113. McMahon, K. M. \& Thaxton, C. S. High-density lipoproteins for the systemic delivery of short interfering RNA. Expert Opin. Drug Deliv. 11 231-247 (2014)

114. Kulkarni, J. A. et al. The current landscape of nuclei acid therapeutics. Nat. Nanotechnol. 16, 630-643 (2021).

This review discusses four platform technologies that have enabled the clinical translation of nucleic acid therapeutics: antisense oligonucleotides, ligand-modified small interfering RNA conjugates, lipid nanoparticles and adeno-associated virus vectors.

115. Curtis, N., Sparrow, A., Ghebreyesus, T. A. \& Netea, M. G. Considering BCG vaccination to reduc the impact of COVID-19. Lancet 395, 1545-1546 (2020).

116. Netea, M. G., van der Meer, J. W. \& van Crevel, R. $B C G$ vaccination in health care providers and the protection against COVID-19. J. Clin. Invest. 131 e145545 (2021)

117. Netea, M. G. et al. Trained immunity: a tool for reducing susceptibility to and the severity of SARS-CoV-2 infection. Cell 181, 969-977 (2020).

118. Noval Rivas, M. et al. BCG vaccination history associates with decreased SARS-CoV-2 seroprevalence across a diverse cohort of health care workers. J. Clin. Invest. 131, e145157 (2021).

119. Moorlag, S. J. C. F. M. et al. Safety and COVID-19 symptoms in individuals recently vaccinated with BCG: a retrospective cohort study. Cell Rep. Med. 1 100073 (2020).

120. Giamarellos-Bourboulis, E. J. et al. Activate: randomized clinical trial of BCG vaccination against infection in the elderly. Cell 183, 315-323.e9 (2020). A randomized trial in the elderly shows that BCG vaccination is safe and protects against viral respiratory infections.

121. Medzhitov, R., Schneider, D. S. \& Soares, M. P. Disease tolerance as a defense strategy. Science 335, 936-941 (2012).

122. Hotchkiss, R. S., Monneret, G. \& Payen, D. Sepsis-induced immunosuppression: from cellular dysfunctions to immunotherapy. Nat Rev Immunol. 13, 862-874 (2013)

123. Winters, B. D. et al. Long-term mortality and quality of life in sepsis: a systematic review. Crit. Care Med. 38, 1276-1283 (2010).

124. Boomer, J. S. et al. Immunosuppression in patients who die of sepsis and multiple organ failure. JAMA 306, 2594 (2011)

125. Domínguez-Andrês, J. et al. The itaconate pathway is a central regulatory node linking innate immune tolerance and trained immunity. Cell Metab. 29, 211-220.e5 (2019).

126. Dunn, G. P., Bruce, A. T., Ikeda, H., Old, L. J. \& Schreiber, R. D. Cancer immunoediting: from immunosurveillance to tumor escape. Nat. Immunol. 3, 991-998 (2002).

127. Dunn, G. P., Old, L. J. \& Schreiber, R. D. The three Es of cancer immunoediting. Annu. Rev. Immunol. 22, 329-360 (2004)

128. Beatty, G. L. \& Gladney, W. L. Immune escape mechanisms as a guide for cancer immunotherapy. Clin. Cancer Res. 21, 687-692 (2015).

129. Wei, S. C., Duffy, C. R. \& Allison, J. P. Fundamental mechanisms of immune checkpoint blockade therapy. Cancer Discov. 8, 1069-1086 (2018).

130. Demaria, O. et al. Harnessing innate immunity in cancer therapy. Nature 574 45-56 (2019).

131. Kondratova, M. et al. A multiscale signalling network map of innate immune response in cancer reveals cell heterogeneity signatures. Nat. Commun. 10, 4808 (2019).

132. McAllister, S. S. \& Weinberg, R. A. The tumourinduced systemic environment as a critical regulator of cancer progression and metastasis. Nat. Cell Biol. 16 717-727 (2014)

133. Casbon, A.-J. et al. Invasive breast cancer reprograms early myeloid differentiation in the bone marrow to generate immunosuppressive neutrophils. Proc. Natl Acad. Sci. USA 112, E566-E575 (2015).

134. Netea, M. G., Joosten, L. A. B. \& van der Meer, J. W. M. Hypothesis: stimulation of trained immunity as adjunctive immunotherapy in cancer. J. Leukoc. Biol. 102, 1323-1332 (2017).

135. Buffen, K. et al. Autophagy controls BCG-induced trained immunity and the response to intravesical BCG therapy for bladder cancer. PLoS Pathog. 10 , e1004485 (2014).

136. Coffman, R. L., Sher, A. \& Seder, R. A. Vaccine adjuvants: putting innate immunity to work. Immunity 33, 492-503 (2010)

137. Jennings, V. A. et al. Potentiating oncolytic virus-induced immune-mediated tumor cell killing using histone deacetylase inhibition. Mol. Ther. 27, 1139-1152 (2019)

138. Bekkering, S. et al. Innate immune cell activation and epigenetic remodeling in symptomatic and asymptomatic atherosclerosis in humans in vivo. Atherosclerosis 254, 228-236 (2016).

139. Bekkering, S. et al. Treatment with statins does not revert trained immunity in patients with familial hypercholesterolemia. Cell Metab. 30, 1-2 (2019).

140. van Leent, M. M. T. et al. Prosaposin mediates inflammation in atherosclerosis. Sci. Transl Med. 13 eabe1433 (2021).

141. Duivenvoorden, R. et al. Nanoimmunotherapy to treat ischaemic heart disease. Nat. Rev. Cardiol. 16 21-32 (2019).

142. Wendeln, A.-C et al. Innate immune memory in the brain shapes neurological disease hallmarks. Nature 556, 332-338 (2018)

143. Crișan, T. O. et al. Soluble uric acid primes TLR-induced proinflammatory cytokine production by human primary cells via inhibition of IL-1 Ra. Ann. Rheum. Dis. 75, 755-762 (2016).

144. Arts, R. J. W., Joosten, L. A. B. \& Netea, M. G. The potential role of trained immunity in autoimmune and autoinflammatory disorders. Front. Immunol 9, 6-9 (2018).

145. Krienke, C. et al. A noninflammatory mRNA vaccine for treatment of experimental autoimmune encephalomyelitis. Science 371, 145-153 (2021).

146. Oberbarnscheidt, M. H. et al. Non-self recognition by monocytes initiates allograft rejection. J. Clin . Invest. 124, 3579-3589 (2014)

147. Netea, M. G. et al. A guiding map for inflammation. Nat. Immunol. 18, 826-831 (2017).

148. Panizzi, P. et al. Impaired infarct healing in atherosclerotic mice with Ly-6ChiMonocytosis. J. Am. Coll. Cardiol. 55, 1629-1638 (2010).

149. Anzai, A. et al. The infarcted myocardium solicits GM-CSF for the detrimental oversupply of inflammatory leukocytes. J. Exp. Med. 214, 3293-3310 (2017). 
150. Swirski, F. K. \& Nahrendorf, M. Cardioimmunology: the immune system in cardiac homeostasis and disease. Nat. Rev. Immunol. 18, 733-744 (2018).

151. Kwong, J. C. et al. Acute myocardial infarction after laboratory-confirmed influenza infection. N. Engl. J. Med. 378, 345-353 (2018).

152. Ridker, P. M. et al. Antiinflammatory therapy with canakinumab for atherosclerotic disease. N. Engl. J. Med. 377, 1119-1131 (2017).

153. Rothlin, C. V. \& Ghosh, S. Lifting the innate immune barriers to antitumor immunity. J. Immunother. Cancer 8, 695 (2020).

154. Goldberg, M. S. Improving cancer immunotherapy through nanotechnology. Nat. Rev. Cancer 19 , 587-602 (2019)

155. Nam, J. et al. Cancer nanomedicine for combination cancer immunotherapy. Nat. Rev. Mater. 4, 398-414 (2019).

156. Shi, Y. $\&$ Lammers, T. Combining nanomedicine and immunotherapy. Acc. Chem. Res. 52, 1543-1554 (2019).

157. Netea, M. G., Quintin, J. \& van der Meer, J. W. M. Trained immunity: a memory for innate host defense. Cell Host Microbe 9, 355-361 (2011).

158. Kinnear, C., Moore, T. L., Rodriguez-Lorenzo, L., Rothen-Rutishauser, B. \& Petri-Fink, A. Form follows function: nanoparticle shape and its implications for nanomedicine. Chem. Rev. 117, 11476-11521 (2017).

159. Hinde, E. et al. Pair correlation microscopy reveals the role of nanoparticle shape in intracellular transport and site of drug release. Nat. Nanotechnol. 12 81-89 (2017)

160. Jiang, Y. et al. The interplay of size and surface functionality on the cellular uptake of sub-10 nm gold nanoparticles. ACS Nano 9, 9986-9993 (2015).

161. Zhao, J. \& Stenzel, M. H. Entry of nanoparticles into cells: the importance of nanoparticle properties. Polym. Chem. 9, 259-272 (2018).

162. Hadjidemetriou, M. \& Kostarelos, K. Nanomedicine: evolution of the nanoparticle corona. Nat. Nanotechnol. 12, 288-290 (2017).

163. Tang, J. et al. Immune cell screening of a nanoparticle library improves atherosclerosis therapy. Proc. Natl Acad. Sci. USA 113, E6731-E6740 (2016).

164. Pérez-Medina, C., Teunissen, A. J. P., Kluza, E., Mulder, W. J. M. \& van der Meel, R. Nuclear imaging approaches facilitating nanomedicine translation. Adv. Drug Deliv. Rev. 154-155, 123-141 (2020).

165. Hu, Z., Chen, W. H., Tian, J. \& Cheng, Z. NIRF nanoprobes for cancer molecular imaging: approaching clinic. Trends Mol. Med. 26, 469-482 (2020).

166. Miller, M. A. \& Weissleder, R. Imaging the pharmacology of nanomaterials by intravital microscopy: toward understanding their biological behavior. Adv. Drug Deliv. Rev. 113, 61-86 (2017)

167. Mulder, W. J. M. et al. MR molecular imaging and fluorescence microscopy for identification of activated tumor endothelium using a bimodal lipidic nanoparticle. FASEB J. 19, 2008-2010 (2005).

168. Yang, Y. S. S. et al. High-throughput quantitation of inorganic nanoparticle biodistribution at the single-cel level using mass cytometry. Nat. Commun. 8, 1-11 (2017).

169. Song, W. et al. Bioanalysis of targeted nanoparticles in monkey plasma via LC-MS/MS. Anal. Chem. 91, 13874-13882 (2019).
170. Zhu, C., Preissl, S. \& Ren, B. Single-cell multimodal omics: the power of many. Nat. Methods 17, 11-14 (2020).

171. Yamankurt, G. et al. Exploration of the nanomedicinedesign space with high-throughput screening and machine learning. Nat. Biomed. Eng. 3, 318-327 (2019).

172. Kedmi, R. et al. A modular platform for targeted RNA therapeutics. Nat. Nanotechnol. 13, 214-219 (2018).

173. Ball, P. The lightning-fast quest for COVID vaccines and what it means for other diseases. Nature 589. 16-18 (2021)

174. Martínez, A. et al. TLR2 and dectin-1 signaling in mouse hematopoietic stem and progenitor cells impacts the ability of the antigen presenting cells they produce to activate CD4 T cells. Cells 9, 1317 (2020).

175. Ifrim, D. C. et al. Trained immunity or tolerance: opposing functional programs induced in human monocytes after engagement of various pattern recognition receptors. Clin. Vaccine Immunol. 21, 534-545 (2014).

176. Mourits, V. P. et al. Lysine methyltransferase G9a is an important modulator of trained immunity. Clin. Transl. Immunol. 10, e1253 (2021)

177. Nahrendorf, M. et al. Hybrid in vivo FMT-CT imaging of protease activity in atherosclerosis with customized nanosensors. Arterioscler. Thromb. Vasc. Biol. 29 1444-1451 (2009).

178. van't Wout, J. W., Poell, R. \& van Furth, R. The role of BCG/PPD-activated macrophages in resistance agains systemic candidiasis in mice. Scand. J. Immunol. 36, 713-719 (1992).

179. Tribouley, J., Tribouley-Duret, J. \& Appriou, M. Effect of Bacillus Callmette Guerin (BCG) on the receptivity of nude mice to Schistosoma mansoni [French] C. R. Seances Soc. Biol. Fil. 172, 902-904 (1978)

180. Holmgren, I. Employment of B.C.G., especially in intravenous injection. Acta Med. Scand. 90, 350-361 (1936).

181. Alexandroff, A. B., Jackson, A. M., O'Donnell, M. A. \& James, K. BCG immunotherapy of bladder cancer: 20 years on Lancet 353, 1689-1694 (1999).

182. Herr, H. W. \& Morales, A. History of bacillus Calmette-Guérin and bladder cancer: an immunotherapy success story. J. Urol. 179, 53-56 (2008).

This article discusses the history of the BCG vaccine and how it became the standard therapy for bladder cancer.

183. Talat lqbal, N. \& Hussain, R. Non-specific immunity of BCG vaccine: a perspective of BCG immunotherapy. Trials Vaccinol. 3, 143-149 (2014).

184. Xiang, B., Snook, A. E., Magee, M. S. \& Waldman, S. A. Colorectal cancer immunotherapy. Discov. Med. 15, 301-308 (2013)

185. Guinan, P., Toronchi, E., Shaw, M., Crispin, R. \& Sharifi, R. Bacillus Calmette-Guérin (BCG) adjuvant therapy in stage D prostate cancer. Urology 20 401-403 (1982)

186. Heyn, R. M. et al. BCG in the treatment of acute lymphocytic leukemia. Blood 46, 431-442 (1975)

187. Schultze, J. L. \& Aschenbrenner, A. C. COVID-19 and the human innate immune system. Cell 184 , 1671-1692 (2021).

188. Tarancón, R. et al. New live attenuated tuberculosis vaccine MTBVAC induces trained immunity and confers protection against experimental lethal pneumonia. PLOS Pathog. 16, e1008404 (2020).

189. Rieckmann, A. et al. Vaccinations against smallpox and tuberculosis are associated with better long-term survival: a Danish case-cohort study 1971-2010. Int. J. Epidemiol. 46, 695-705 (2016)

190. Aaby, P. et al. Non-specific beneficial effect of measles immunisation: analysis of mortality studies from developing countries. BMJ 311, 481-485 (1995).

191. Andersen, A. et al. National immunization campaigns with oral polio vaccine reduce all-cause mortality: a natural experiment within seven randomized trials. Front. Public Heal. 6, 13 (2018)

192. Benn, C. S., Netea, M. G., Selin, L. K. \& Aaby, P. A small jab - a big effect: nonspecific immunomodulation by vaccines. Trends Immunol. 34, 431-439 (2013).

193. Zhao, Y. et al. Near-infrared fluorescence energy transfer imaging of nanoparticle accumulation and dissociation kinetics in tumor-bearing mice. ACS Nano 7, 10362-10370 (2013)

194. Sanchez-Gaytan, B. L. et al. Real-time monitoring of nanoparticle formation by FRET imaging Angew. Chem. Int. Ed. 56, 2923-2926 (2017).

195. Lieberman, B. P. et al. PET imaging of glutaminolysis in tumors by ${ }^{18} \mathrm{~F}-(2 S, 4 R) 4-$ fluoroglutamine. J. Nucl. Med. 52, 1947-1955 (2011)

196. Yeh, H.-H. et al. Imaging epigenetic regulation by histone deacetylases in the brain using PET/MRI with ${ }^{18}$ F-FAHA. Neuroimage 64, 630-639 (2013).

197. Ye, Y.-X. et al. Imaging macrophage and hematopoietic progenitor proliferation in atherosclerosis. Circ. Res. 117, 835-845 (2015)

198. Rashidian, M. et al. Immuno-PET identifies the myeloid compartment as a key contributor to the outcome of the antitumor response under PD-1 blockade. Proc. Natl Acad. Sci. USA 116, 16971-16980 (2019).

199. Teunissen, A. J. P. et al. Employing nanobodies for immune landscape profiling by PET imaging in mice. STAR Protoc. 2, 100434 (2021)

200. Kunjachan, S. et al. Noninvasive optical imaging of nanomedicine biodistribution. ACS Nano 7, 252-262 (2013).

\section{Acknowledgements}

This work was supported by National Institutes of Health (NIH) grants R01 HL144072, R01 CA220234, and P01 HL131478, as well as a Vici grant from the Dutch Research Council NWO and a European Research Council (ERC) Advanced Grant (no. 101019807) (all to W.J.M.M.). M.G.N. was supported by a Spinoza grant from the Netherlands Organization for Scientific Research and an ERC Advanced Grant (no. 833247)

\section{Author contributions}

M.M.T.v.L., B.P. and W.J.M.M. wrote the manuscript. All authors researched data for the article, provided substantial contributions to discussion of content and reviewed and edited the manuscript before submission.

\section{Competing interests}

W.J.M.M. and M.G.N. are scientific founders and shareholders of Trained Therapeutix Discovery.

\section{Peer review information}

Nature Reviews Materials thanks Angus Johnston and the other, anonymous, reviewers for their contribution to the peer review of this work.

Publisher's note

Springer Nature remains neutral with regard to jurisdictional claims in published maps and institutional affiliations.

(C) Springer Nature Limited 2022 